\title{
Crystal rotations and alignment in spatially varying magma flows: 2-D examples of common subvolcanic flow geometries
}

\author{
Rémi Vachon ${ }^{\oplus},{ }^{1}$ Mohsen Bazargan ${ }^{\odot},{ }^{2}$ Christoph F. Hieronymus, ${ }^{2}$ Erika Ronchin ${ }^{3}$ and \\ Bjarne Almqvist ${ }^{2}$ \\ ${ }^{1}$ Centre for Arctic Gas Hydrate, Environment and Climate, Universitetet i Tromsø, Naturfagbygget Dramsveien 201, NO-9010 Tromsø, Norway. E-mail: \\ remi.e.vachon@uit.no \\ ${ }^{2}$ Department of Earth Sciences, Uppsala Universitet, Villavägen 16, SE-75236 Uppsala, Sweden \\ ${ }^{3}$ Department of Earth Sciences, Sapienza University of Rome, 00185 Roma RM, Italy
}

Accepted 2021 March 29. Received 2021 March 22; in original form 2020 August 14

\begin{abstract}
SUMMAR Y
Elongate inclusions immersed in a viscous fluid generally rotate at a rate that is different from the local angular velocity of the flow. Often, a net alignment of the inclusions develops, and the resulting shape preferred orientation of the particle ensemble can then be used as a strain marker that allows reconstruction of the fluid's velocity field. Much of the previous work on the dynamics of flow-induced particle rotations has focused on spatially homogeneous flows with large-scale tectonic deformations as the main application. Recently, the theory has been extended to spatially varying flows, such as magma with embedded crystals moving through a volcanic plumbing system. Additionally, an evolution equation has been introduced for the probability density function of crystal orientations. Here, we apply this new theory to a number of simple, 2-D flow geometries commonly encountered in magmatic intrusions, such as flow from a dyke into a reservoir or from a reservoir into a dyke, flow inside an inflating or deflating reservoir, flow in a dyke with a sharp bend, and thermal convection in a magma chamber. The main purpose is to provide a guide for interpreting field observations and for setting up more complex flow models with embedded crystals. As a general rule, we find that a larger aspect ratio of the embedded crystals causes a more coherent alignment of the crystals, while it has only a minor effect on the geometry of the alignment pattern. Due to various perturbations in the crystal rotation equations that are expected in natural systems, we show that the time-periodic behaviour found in idealized systems is probably short-lived in nature, and the crystal alignment is well described by the time-averaged solution. We also confirm some earlier findings. For example, near channel walls, fluid flow often follows the bounding surface and the resulting simple shear flow causes preferred crystal orientations that are approximately parallel to the boundary. Where pure shear deformation dominates, there is a tendency for crystals to orient themselves in the direction of the greatest tensile strain rate. Where flow impinges on a boundary, for example in an inflating magma chamber or as part of a thermal convection pattern, the stretching component of pure shear aligns with the boundary, and the crystals orient themselves in that direction. In the field, this local pattern may be difficult to distinguish from a boundary-parallel simple shear flow. Pure shear also dominates along the walls of a deflating magma chamber and in places where the flow turns away from the reservoir walls, but in these locations, the preferred crystal orientation is perpendicular to the wall. Overall, we find that our calculated patterns of crystal orientations agree well with results from analogue experiments where similar geometries are available.
\end{abstract}

Key words: Numerical modelling; Probability distributions; Physics of magma and magma bodies. 


\section{INTRODUCTION}

The dynamics of magmatic intrusions and of volcanic eruptions are ultimately controlled by the geometry of the volcanic plumbing system and by the magma flow patterns and flow regimes within the various channels and reservoirs. Remote sensing techniques such as measurement of surface deformations or seismicity can provide information on active volcanic systems, but the resulting picture of the intrusion is typically non-unique and at low spatial resolution, and it generally provides little detail of the channel geometry and the flow pattern (Yun et al. 2006; Montgomery-Brown et al. 2010; Pallister et al. 2010; Nobile et al. 2012). An alternative approach for understanding magma dynamics is to analyse solidified and exposed magmatic systems. In addition to direct observation of the geometry of the intrusion, the solidified magma often contains one or several flow indicators such as magmatic banding and foliation, and frequently also a net alignment of elongate inclusions such as phenocrysts or enclaves (Clemens 2003; Pons et al. 2006; Vernon \& Paterson 2006).

A number of tools are available for measuring shape preferred orientation (SPO). One approach is to identify the orientation of individual elongate inclusions such as crystals or enclaves, which can be done by imaging techniques such as microscopy (Launeau \& Robin 1996; Launeau \& Cruden 1998) or X-ray microcomputer tomography (micro-xCT, Cnudde et al. 2011; Zucali et al. 2014; Petri et al. 2020). Whereas direct imaging techniques are restricted to 2-D cross sections, tomographic analysis allows determination of the inclusion shape and orientation in three dimensions. The micro$\mathrm{xCT}$ method is to some extent limited by the attenuation contrast between minerals, which depends mainly on the density contrast between the minerals that constitute the rock. Minerals with density similar to their surroundings are thus difficult to image. In addition, the micro-xCT analysis is both computationally and labor intensive, which in its present state limits its application to relatively small volumes of investigated material. An alternative approach is based on measurement of anisotropy of magnetic susceptibility (AMS) of rock samples, which provides a statistical estimate of crystal alignment (Tarling \& Hrouda 1993; Borradaile \& Jackson 2004). Magnetic susceptibility is a symmetric secondrank tensor which can be represented as an ellipsoid in 3-D space. AMS is in some cases directly linked with SPO, as in foliated and lineated metamorphic rocks with the magnetic signal dominated by the paramagnetic fraction (Khan 1962; Silva et al. 2001, and references therein), although the details on how to interpret their relationship remains a challenge (Knight \& Walker 1988; Geoffroy et al. 2002). The main advantages of the AMS method are its high measurement sensitivity and its ability to process large quantities of data in relatively short time. As a result, AMS is routinely used to determine flow directions in magmatic systems (Henry 1997; Tauxe et al. 1998; Silva et al. 2004; Cañón-Tapia \& ChávezAlvarez 2004; Cañón-Tapia 2004; Palmer et al. 2007; Silva et al. 2010, 2014; Eriksson et al. 2011). Special care must be taken when comparing AMS with SPO, especially when the magnetic signal is dominated by the ferrimagnetic fraction, as in mafic dykes (Silva et al. 2014). In this case, ferrimagnetic and paramagnetic SPOs are not coaxial, and the angular difference between the ferrimagnetic and paramagnetic fabrics can be high. Given the increasing availability of SPO data, and in particular of spatially extensive AMS data and the high potential of $\mathrm{xCT}$ microtomography to directly infer the crystal orientation, it is timely to improve our analytical abilities of linking the SPO observations with the magma flow field.
To date, most analyses of crystal orientations have been largely inferential. Observations of crystal orientation distributions in solidified dykes show that elongate particles are usually oriented subparallel to the dyke walls, and the magma flow direction is assumed to be approximately aligned with the long particle axis (Smith 2002; Paterson 2009; Chistyakova \& Latypov 2010; Yamato et al. 2011). The alignment of crystals and magnetic fabric in laccoliths, on the other hand, is believed to be caused by magma flowing radially outward from the centre of the main body of the laccolith (Pons et al. 2006; Mattsson et al. 2018). For a sill with fingerlike lobes, Horsman et al. (2005) estimate the direction of magma flow from observations of magnetic lineation and crystal alignment. They show that the fabric is mostly subparallel to the bounding surfaces of the flow, and infer that the flow is largely parallel to the lineations.

Systematic interpretation of the observed fabric of inclusion orientations is only possible if a rigorous model is available that allows prediction of inclusion orientations based on the details of the surrounding flow. Analogue simulations using a viscous fluid with embedded elongate particles provide one such model. Závada et al. $(2009,2015)$ use analogue modelling coupled with AMS analysis of magnetite tracers to show that the preferred crystal orientation is generally aligned with the direction of the greatest tensile principal strain near the centre of a spreading flow. However, near the boundaries of a lava dome as well as along the walls of its feeder conduit, the fabric is mostly aligned parallel to the boundaries. Trebbin et al. (2013) use analogue modelling with immersed polymer micelles to follow the particle orientation associated with the flow field in a pipe system with a local constriction. They find that the micelles are aligned parallel to the flow direction in the first part of the pipe, and retain this orientation during the transition and throughout the narrow portion of the pipe. As the flow passes the expanding portion of the pipe, however, the micelles get reoriented and align perpendicular to the flow direction.

Numerical modelling of crystal rotations in viscous flows provides an alternative to analogue models. The fundamental advantages of analytical or numerical models is that results are given in quantitative form, and that model parameters, such as viscosity, and their functional dependence on the different flow variables can be selected freely, while analogue models are contingent on the availability of suitable materials. The analytical solution for the rotation of an isolated ellipsoidal particle in a viscous flow (Jeffery 1922) forms the basis for most calculations. Numerous studies have utilized this solution to calculate the evolution of the orientation of individual crystals in homogeneous flows (i.e. flows with spatially constant strain rate, Freeman 1985; Passchier 1987; Ježek et al. 1994; Marques et al. 2005; Jiang 2007, 2012; Marques et al. 2014). It has been shown that the solution for an isolated ellipsoid is approximately valid also for suspensions of multiple particles, provided that the interparticle spacing corresponds to at least 12 particle lengths (Ildefonse et al. 1992a,b). Probability density functions (PDF) of crystal orientations can then be computed for certain crystal geometries based on the finite strain ellipsoid (March 1932; Willis 1977), or, for more general crystal shapes, by explicitly evaluating the rotations of a large ensemble of crystals (March 1932; Reed \& Tryggvason 1974; Willis 1977; Ježek et al. 1996; Yamato et al. 2011). Bazargan et al. (2019) introduced an evolution equation for the PDF of crystal orientations based on the velocity field of the fluid flow which allows the PDF to be computed for any crystal shape for which the individual rotation behaviour is known (either analytically or numerically). 
In Earth sciences, the analysis of particle rotations in viscous fluids has been limited to homogeneous flows. This restriction poses little problem for applications to tectonically deformed regions where strain rates tend to be constant over large distances and over long timescales. However, other flows, such as magma transport in subsurface channels, exhibit much greater variability in space and time, and an analysis in terms of homogeneous deformations is overly restrictive. In other branches of fluid mechanics, the theory of inclusion rotations has been applied successfully to systems with variable flows (Szeri 1993; Fries et al. 2017). This approach is valid provided that the length scale over which the fluid velocity varies is greater than the crystal dimensions (Jeffery 1922; Ildefonse et al. 1992b; Mandal et al. 2003; Kumar Samanta et al. 2003). The large-scale flow is then found by solution of the Navier-Stokes equation for the fluid without inclusions, and the PDF of crystal orientations is evaluated simultaneously by coupling the evolution equation (Bazargan et al. 2019) with the velocity solution. In the present study, we use this technique for a number of relatively simple, 2-D fluid systems, each of which is representative of a flow pattern that may be considered typical of magmatic intrusions. Despite their simple geometry, the flow fields are sufficiently complex to require numerical solution. The computed PDFs are 4-D (two spatial dimensions, one orientation angle and time). The general theory of computing the PDF is also valid for 3-D flows and ellipsoidal inclusions with three distinct axis lengths, but the resulting 7-D problem (three spatial dimensions, three orientation angles, and time) is computationally expensive and is left for future studies.

The purpose of the present study is to explore the type of alignment patterns that result from different 2-D flow geometries, and to compare these results with analog models in order to test our theory and numerical method. A number of simplifying assumptions were made in order to minimize the number of free parameters. The magma viscosity is taken to be constant. The geometry of the magma plumbing system is either fixed or explicitly prescribed so that host rock deformations do not need to be modelled. The suspended crystals are elliptical and have identical aspect ratios. Clearly, nature is generally more complex. However, for model validation, our simple models are most appropriate because analog models are generally based on similar assumptions. For more detailed case studies, the problem to be solved is typically the inverse of our models; that is, the crystal alignment pattern is observed, and an appropriate flow field has to be found which produces this pattern. Our simple models can function as a starting point for such case studies.

\section{METHODS}

For most of our models, the geometry of the magma plumbing system is constant in time. For the two models with changing geometry (inflating and deflating magma reservoir), the deformation of the magma-host rock interface is prescribed. Hence, the host rock is not modelled explicitly in our study.

In all models, the viscosity is taken to be constant. This choice was made in order to avoid complications due to magma composition, due to the mathematical formulation of the temperature-viscosity function, and due to the magma flow rate which would affect the cooling rate. Thermal buoyancy is neglected in all models except for the thermally convecting magma reservoir, and with the exception of this one model, the temperature field is not computed.

For the magma flow, we assume that the viscosity is sufficiently large (i.e. low Reynolds number) so that inertial effects are negligible. The dynamics are then governed by the Navier-Stokes equation in the limit of Stokes flow (Batchelor 2000),

$-\nabla p+\nabla \cdot(\mu \nabla \mathbf{v})+\rho \mathbf{g}=0$,

where $p$ is the pressure in the magma, $\mu$ is the magma viscosity, $\mathbf{v}$ is the velocity vector, $\rho$ is the density and $\mathbf{g}$ is the acceleration due to gravity. The flows examined in the present study are 2-D, hence the gradient operator in Cartesian coordinates is given by $\nabla=\hat{\mathbf{x}} \partial / \partial x+\hat{\mathbf{y}} \partial / \partial y$. All flow systems are assumed to be incompressible, and thus the continuity equation becomes (Batchelor 2000)

$\nabla \cdot \mathbf{v}=0$

In the example of thermal convection, the flow is driven by density differences due to temperature, which are described by the last term on the left-hand side of (1). For all other flows, density is assumed constant so that the buoyancy term has no dynamic effect. For the thermal convection calculations, we use the Boussinesq approximation (Batchelor 2000) which allows us to use the incompressible form of the continuity eq. (2) together with the energy equation,

$\frac{\partial T}{\partial t}+\mathbf{v} \cdot \nabla T=\kappa \nabla^{2} T$.

Here $T$ is temperature and $\kappa$ is the thermal diffusivity, which is assumed to be constant. For the convection computations, density is taken to be linearly dependent on temperature, that is

$\rho(T)=\rho[1-\alpha(T-T)]$,

where $\rho$ is the density at the reference temperature $T=T$, and $\alpha$ is the volumetric thermal expansion coefficient.

Rotations are calculated for 2-D elliptical crystals with motions and spin limited to the plane of the fluid flow. Rather than determining the rotational evolution for individual crystals, we compute the PDF orientations based on the theory developed by Bazargan et al. (2019),

$\frac{\partial P}{\partial t}+\mathbf{v} \cdot \nabla P=-P \frac{\partial \dot{\theta}}{\partial \theta}-\dot{\theta} \frac{\partial P}{\partial \theta}$.

Here $P$ is the value of the PDF, which at every point in space and time is a function of the crystal orientation $\theta$, which in turn is the angle between the $x$-axis and the crystal's semi-major axis. The crystal rotation rate $\dot{\theta}$ is calculated using the framework of Jeffery (1922), which for a 2-D elliptical crystal in a fixed reference frame can be written as

$\dot{\theta}=w_{x y}-\frac{\left(\dot{\varepsilon}_{1}-\dot{\varepsilon}_{2}\right)}{2} \frac{\left(a^{2}-b^{2}\right)}{\left(a^{2}+b^{2}\right)} \sin \left(2 \theta-2 \theta_{p}\right)$,

where $w_{x y}=\frac{1}{2}(\partial v / \partial x-\partial u / \partial y)$ is the local value of the fluid's angular velocity, $\dot{\varepsilon}_{1}$ and $\dot{\varepsilon}_{2}$ are the local principal strain rates of the flow (ordered such that $\dot{\varepsilon}_{1} \geq \dot{\varepsilon}_{2}$ ), $\theta_{p}$ is the angle between the $x$-axis and the direction of $\dot{\varepsilon}_{1}$ and $a$ and $b$ are the semi-major and semi-minor axes of the crystal, respectively. The value of $w_{x y}$ locally describes the component of solid body rotation of the flow, while the values of $\dot{\varepsilon}_{1}$ and $\dot{\varepsilon}_{2}$ together describe the deformation due to pure shear.

The coupled system of the fluid mechanical equations together with the evolution equation for the PDF field $P$ is solved using the finite element method on an Eulerian grid. The solution is computed using the finite element package Comsol Multiphysics (Comsol Multiphysics R 2017). We show results for four different flow geometries: (i) flow from a channel into a reservoir, (ii) an inflating circular magma chamber, (iii) flow through a dyke with a sharp bend and (iv) thermal convection in a rectangular domain. Except 
for the convection system, the flow directions can be reversed so that we can use the same model geometries to compute the effects of a flow from a reservoir into a channel, and of flow inside a deflating magma chamber. As the crystal rotation patterns depend not only on the local values of the solid body rotation and the pure shear but also on the advection of the crystals in the flow, the effect of a reversal of the flow direction on the crystal rotation behaviour is not trivial.

For all models of this study, the flow field is 2-D. For most computations, we use an unstructured mesh with triangular elements in $x-y$-space, while for the convection model (in a rectangular box), we use a regular mesh of quadrilateral elements. The PDF of crystal orientations is computed at the same locations as the flow velocities. However, as the PDF describes the probability density as a function of crystal orientation $\theta$, the PDF is a 3-D field. While the geometries and the mesh resolution vary from model to model, the $\theta$-dimension of all dimensions is a constant range. We thus use a regular mesh spacing in the $\theta$-direction, with 20 elements for most models (resolution of 0.157 radians), except for the convection model with 32 elements (resolution of 0.0982 radians). We use linear elements for the calculations of the PDF function $P$ and for temperature in the convection models, but in the fluid flow calculations, we use a combination of quadratic integration for the velocity field coupled with linear integration for pressure. Due to rotational symmetry of the crystals over half of a revolution, crystal orientations only need to be computed in the $\theta$-range of $[0, \pi]$. Rotations beyond these limits are handled by periodic boundary conditions in $\theta$. We have tested the models with twice the resolution both in physical ( $x$ and $y$ ) space, and for the PDF also in $\theta$-space. We found only negligible sensitivity to doubling the resolution, and therefore conclude that the chosen resolution is sufficient for the given flow fields.

In a previous study (Bazargan et al. 2019), PDFs of crystal orientations were calculated for a number of flows with analytical solutions for the flow field. There is one significant difference in the two techniques used. In the previous study, the velocity field was integrated to yield the positions $x(t)$ and $y(t)$ along the paths of a number of selected fluid parcels. The time rates of solid body rotation and of pure shear deformation were then computed analytically along the path. Most importantly, the Lagrangian approach of following fluid parcels avoids the explicit calculation of the advection terms in (5). In this study, we use the more standard approach in fluid mechanics which computes all quantities on a fixed, Eulerian grid. The advection terms in (5) are then retained. The semianalytical solutions from Bazargan et al. (2019) for Couette flow, for plane Poiseuille flow, and for corner flow can then be used as a benchmark for testing the fully numerical results. When the numerical solution converges properly (i.e. the time steps are sufficiently small such that the fluid covers less than about one quarter of an element length per time increment, thus ensuring that no oscillations develop in the advected field), we find that the solutions are essentially identical. We thus conclude that the fully numerical solution is satisfactory.

\subsection{Transition between channel and reservoir}

Magma flow from a planar channel (i.e. a dyke) into a larger reservoir such as a magma chamber or a laccolith is a common feature in volcanic plumbing systems. Similarly, magma frequently withdraws from a reservoir via a planar dyke. We set up a suitable geometry with imposed inflows and outflows to study these phenomena in terms of the rotational behaviour of suspended crystals. For both the inflow and the outflow model, the geometries are equal with a rectangular reservoir that is 50 by $10 \mathrm{~m}$ in size, and which at the centre of one of the $10 \mathrm{~m}$-bounding walls is connected to a channel that is $5 \mathrm{~m}$ long and $1 \mathrm{~m}$ in thickness. The channel is long enough so that, after passage, the flow is fully developed (i.e. Poiseuille flow), and the crystal orientations have reached their long-term behaviour which is not steady, but rather a periodic, oscillatory state (Bazargan et al. 2019). The reservoir has an outflow (or inflow) at the side opposite the channel which exactly balances the flow rate specified for the channel. The long reservoir dimension of $50 \mathrm{~m}$ is sufficient to make sure that, in the vicinity of the dyke-reservoir transition, there are no flow perturbations due to the distal boundary. At the connection between reservoir and channel, we tested transitions with sharp corners and with rounded corners. We found only minimal differences, and here we show the results for the rounded corners (the domain geometry is not shown separately, but can be seen in the plots of the results). The flow velocity at the end of the channel is set to an arbitrary constant value of $10 \mathrm{~m} \mathrm{~s}^{-1}$, which, due to the incompressibility condition of the fluid, must be balanced by a corresponding velocity of $1 \mathrm{~m} \mathrm{~s}^{-1}$ at the opposite end of the reservoir. The absolute values of the model size and magma velocities are irrelevant, as the models can be scaled using any other length and velocity scales. The only significant choice in our models is the ratio of the inlet size to the size of the reservoir, which sets the relative lengths as well as the relative velocities. Since the inertial terms are neglected in the Navier-Stokes eq. (1), high velocities do not lead to turbulence or other inertial effects, and the solution is not affected by different choices of density or viscosity. Furthermore, the development of the crystal orientation fabric depends on the total strain, but not on how fast this strain develops, thus the PDF pattern depends only on the flow pattern, but not on the length or viscosity scales. The PDF is set to a uniform distribution (a constant value of $1 / \pi)$ as initial condition, and is maintained at the same value at the respective inlet throughout the model run. For computation of the viscous flow and for the PDF, the spatial (i.e. $x-y$ ) domain is discretized using 6548 triangular elements, giving an approximate element size of $0.3 \mathrm{~m}$. Given the quadratic discretization of the velocity solution, the resolution corresponds to seven nodes across the channel, which is adequate to image the Poiseuille flow conditions. The model is run for a total time of $t_{\text {total }}=400 \mathrm{~s}$.

\subsection{Inflating or deflating magma reservoir}

The formation and evolution of magma reservoirs is complex and probably involves a number of different mechanisms. In dynamic models, the volumetric growth is often modelled assuming a purely elastic response of the surrounding host rock (Gudmundsson 2012), even though the resulting stresses may surpass the failure strength of the host rock. Fracturing of the wall rock may lead to local concentration of strains, or, if broadly distributed and pervasive, may produce deformations of the reservoir walls that are similar to those of the elastic model. Given the frequent use of the elastic models as well as their simplicity, we take a similar approach here, albeit without explicit reference to the host rock rheology. In our model of an inflating reservoir, a circular chamber (i.e. cylindrical in $3-D)$ is inflated at a constant rate of volume increase from an initial radius of $1 \mathrm{~m}$ to a final radius of $2 \mathrm{~m}$. The volumetric inflation is balanced by a stress-free inlet that spans a constant 0.28 radians of the wall of the circular chamber. The imposed uniform extension of the chamber walls is approximately what would be expected if 
magma were injected into a chamber placed in an infinite space of elastic material. Since the model domain changes as a function of time, we use an arbitrary Lagrangian-Eulerian framework (ALE, Comsol Multiphysics@ 2017). At the boundaries, the mesh follows the magma, and the approach may locally be described as Lagrangian. Elsewhere, however, the mesh stretches broadly to follow the boundaries while at the same time maintaining an even distribution of element sizes with elements of approximately equilateral shape. The mesh thus deforms, but is independent of the local fluid displacements. The $x-y$ domain is discretized using a total of 4400 triangular elements, giving a spatial resolution of about $4 \times 10^{-2} \mathrm{~m}$. The initial condition for the PDF of crystal orientations is a uniform distribution with a value of $P=1 / \pi$. The same uniform distribution is maintained at the inlet throughout the model run.

\subsection{Flow inside a channel with a sharp bend}

Owing to their formation by fracture mechanical processes, dykes and sills often follow crooked paths with sharp kinks and sudden changes in direction. Occasionally, one also finds field examples of dykes entering sills in a T-shaped junction (Galland et al. 2018). We set up three different dyke geometries with imposed in- and outflow conditions to test their effect on the preferred orientation of suspended particles. The first two models comprise a dyke with constant thickness and with an abrupt change in direction of $\alpha=$ $45^{\circ}$ and $\alpha=90^{\circ}$, respectively. The corners of the bend are not rounded but sharp. In the third model, magma flows from a vertical dyke via a T-shaped junction into a horizontal sill. We chose the channel thicknesses such that the average velocities are the same everywhere, that is, the vertical dyke has twice the thickness of the horizontal sill. As in the example of the kinked dyke, the transition in the channel geometry is sharp, that is the radius of curvature at the joint is zero. For all models, channel thickness is set to a constant value of $2 \mathrm{~m}$ throughout ( $1 \mathrm{~m}$ for the horizontal sill in the T-shaped junction), and the flow velocity at the inlet is set to a constant value of $1 \mathrm{~m} \mathrm{~s}^{-1}$. The initial condition for the PDF as well as the condition at the inlet is a uniform distribution with value $P=1 / \pi$. The flow plane is discretized using 1814 triangular elements ( 10280 elements for the dyke-to-sill transition), giving a spatial resolution of about $0.2 \mathrm{~m}$. The model is run for a total time of $t_{\text {total }}=200 \mathrm{~s}$.

\subsection{Thermal convection in magma reservoir}

The existence and vigor of thermal convection are governed by the Rayleigh number, which is a measure of the strength of the driving forces (i.e. buoyancy) relative to the viscous drag force which counteracts convective motions (Turcotte \& Schubert 2014). Convection in initially uniformly hot magma reservoirs develops by cooling of magma against the roof of the reservoir (Marsh 1989), and the increasing thickness of the boundary layer increases the system's Rayleigh number. Convection in a magma reservoir thus starts from the top, but rapidly spreads throughout the entire domain. Based on thermo-mechanical modelling, convection is expected to occur for most magma types (even those with relatively high viscosity) for magma bodies with heights of $10-100$ s of metres. Thermal convection is thus expected to be a common feature of magmatic systems, and it is therefore important to be able to interpret the observed crystal orientations accordingly.

In our model, we focus on a simple convective system in a rectangular box with constant high temperature maintained at the bottom
Table 1. Fluid and thermal properties of the material for the convection model, with $\mu$ the viscosity, $\rho_{\mathrm{o}}$ the reference density, $\alpha$ the volumetric expansion coefficient, $k$ the thermal conductivity, $c_{p}$ the specific heat at constant pressure and $\Delta T$ the temperature difference between the base and the top of the reservoir.

\begin{tabular}{lcc}
\hline Parameters & Values & Units \\
\hline$\mu$ & $1 \times 10^{7}$ & Pa.s \\
$\rho_{0}$ & 2800 & $\mathrm{~kg} \mathrm{~m}^{-3}$ \\
$\alpha$ & $3 \times 10^{-5}$ & $1 \mathrm{~K}^{-1}$ \\
$k$ & 2.25 & $\mathrm{~W} \mathrm{~m}^{-1} \mathrm{~K}^{-1}$ \\
$C_{p}$ & 750 & $\mathrm{~m}^{2} \mathrm{~s}^{-2} \mathrm{~K}^{-1}$ \\
$\Delta T$ & 400 & ${ }^{\circ} \mathrm{K}$ \\
\hline
\end{tabular}

surface, and constant low temperature maintained at the top surface. The evidence of convection that can potentially be observed in nature in terms of crystal fabric must generally represent the last increments of flow before convection ceases. The Rayleigh number at this time is near the critical limit. Whether the flow is caused by cooling of the magmatic body or by heating from below, the convective style is characterized by broad features and relatively stable convection cells. We run our convection system with a Rayleigh number of $R a=1400$, which results in stable cells with low velocity and broad boundary layers and up- and downwellings. The appropriate Rayleigh number for bottom-heated convection is given by $R a=\left(g \rho c_{p} \alpha \Delta T D^{3}\right) /(k \mu)$, where $c_{p}$ is the specific heat, $\alpha$ is the volumetric expansion coefficient, $\Delta T$ is the temperature difference between the base and the top of the convective layer, and $D$ is the thickness of the layer. The model is run for a duration that allows several convective overturns. Crystal orientation patterns are observed to get essentially completely overprinted over such strains, so $a$ posteriori it is found that there is no need to run the model for longer time periods.

The model domain is a rectangular box with a width of $200 \mathrm{~m}$ and a height of $50 \mathrm{~m}$, and hence with an aspect ratio of width/height $=4$. At all boundaries, the normal and tangential fluid velocities are set to zero. Temperature at the top and bottom boundaries is set to constant values (the absolute value of $T$ is not important as it is only the value of the Rayleigh number that determines the convective pattern), and the side boundaries are thermally insulated. As there is no fluid flow across the boundaries, the normal flux of the PDF of crystal orientations is zero there, that is $c \hat{\mathbf{n}} \cdot \nabla P=0$, where $\hat{n}$ is the unit normal to the boundary. The fluid and thermal properties of the material are descibed in Table 1. The initial condition for the PDF is a uniform value of $P=1 / \pi$ everywhere. The spatial domain (i.e. $x-y$ ) is discretized using 10251 quadrilateral elements for computation of the viscous flow and 400 quadrilateral elements for the PDF field.

\subsection{Vorticity number}

The vorticity number is defined as the ratio of the solid-body rotation rate to the pure shear stretching rate (Truesdell 1953; Bazargan et al. 2019), or

$W_{k}=\frac{\sqrt{2} \Omega}{\left(\dot{\varepsilon}_{1}^{2}+\dot{\varepsilon}_{2}^{2}+\dot{\varepsilon}_{3}^{2}\right)^{1 / 2}}$,

where $\Omega$ is the magnitude of the angular velocity vector. It is straightforward from the rotation dynamics of a single crystal to see that a crystal will progressively align with the principal axis that corresponds to the greatest strain rate, $\dot{\varepsilon}_{1}$. For a large number of crystals 
immersed in a flow with uniform orientation of the pure shear field, the corresponding PDF of crystal orientations tends towards a Dirac delta function. The solid body rotation generally works against the alignment caused by pure shear because the flow vorticity tends to rotate the existing crystal fabric out of the orientation of the pure shear field. Overall, one may thus expect that the evolution of the strength of the crystal orientation fabric in some way depends on the vorticity number, although the dynamics are more complicated because the crystals are advected through a pure shear field that generally changes direction along their path.

\subsection{Degree of orientation}

The strength of the fabric of crystal orientations may be quantified using a measure termed the degree of orientation (Fisher 1995; Bazargan et al. 2019),

$$
R=\pi\left[\left(\int_{0}^{\pi} P \cos (2 \theta) \mathrm{d} \theta\right)^{2}+\left(\int_{0}^{\pi} P \sin (2 \theta) \mathrm{d} \theta\right)^{2}\right]^{1 / 2} .
$$

The degree of orientation $R$ is zero for a uniform distribution, and it has a maximum value of $R=1$ when the PDF is an appropriately normalized Dirac delta function. Coherent pure shear deformation over a prolonged time interval is probably rarely attained in natural flows, and hence the degree of orientation $R$ usually remains well below unity. For a simple shear flow, the vorticity number is $W_{k}$ $=1$ (Bazargan et al. 2019), and the degree of orientation depends on the crystal shape and aspect ratio. For a 2-D elliptical particle with aspect ratio $a / b=2$, the steady-state PDF in a Couette flow (which is also equal to the time-averaged PDF over the oscillatory behaviour seen in most simple-shear PDF solutions) yields a degree of orientation of $R=0.3$ (Bazargan et al. 2019). Poiseuille flow is pressure-driven flow through a plane channel of constant thickness (Turcotte \& Schubert 2014). Throughout a Poiseuille flow, the vorticity number is $W_{k}=1$, which is the same as for simple shear. Moreover, the orientation of the principal axis corresponding to $\dot{\varepsilon}_{1}$ is $45^{\circ}$ relative to the flow direction in both flows. Averaged over a sufficiently large region, the PDF of crystal orientations in a Poiseuille flow thus results in the same degree of orientation as in the Couette flow, namely $R=0.3$ for a crystal aspect ratio of two. Simple shear and Poiseuille flows comprise the most commonly observed flow types in nature and in analogue experiments. The theoretically predicted value of $R$ for these flows thus provides a useful scale for comparing the numerical results with observations.

\section{RESULTS}

\subsection{Periodic versus non-periodic behaviour of the crystal orientation PDF}

It is well known that elliptical crystals embedded in a Couette flow lead to a time-periodic solution of the orientation PDF if the crystals are initially in a perfectly random state (March 1932; Willis 1977; Bazargan et al. 2019). If this type of behaviour is a general characteristic of elongate crystals in viscous flows, then the instantaneous crystal orientations are merely a snapshot of an everevolving PDF field. On the other hand, it has been shown that the exact same Couette flow also allows for steady rotational behaviour given by a constant orientation PDF, provided that the initial PDF is not uniform, but a particular function that represents an intermediate state of crystal alignment (Bazargan et al. 2019, eq. 23). In this case, the instantaneous PDF field is representative of the entire solution throughout time. For the presentation of the results, it is therefore important to know from the onset whether the PDF field is time-variable (e.g. periodic) or whether it tends towards a steady state.

At any point in time, a given fluid parcel must either have been advected into the model domain via an inlet in the boundary (i.e. 'new fluid'), or else the fluid parcel must have originated in the model domain at beginning of the model run ('old fluid'). Most of our models are steady flows with a fixed geometry which are driven by inflows and outflows. In these models, all fluid is eventually replaced by new fluid (with the exception of points where the fluid velocity is zero). Under these conditions, all fluid parcels passing through a given location in the model domain will have experienced the same flow and the same total integrated strain at the moment at which they occupy that particular location. In other words, the PDF field (which is a function of the integrated strain history) is also in steady state, and hence there is no periodicity in time.

In a numerical model (which can only be run for a finite length of time), steady-state conditions with new fluid at all locations are never attained at or near fixed boundaries or stagnation points where the fluid velocity is zero. For a planar Poiseuille flow, it has been shown previously (Bazargan et al. 2019) that the total strain integrated from the point where the fluid enters via an inlet increases from the centre towards the channel boundaries where the strain reaches infinity. The time-periodic behaviour of the crystal rotations then manifests itself in alternating bands of alignment and disorder, and these bands become infinitesimaly thin near the channel boundaries. When averaged over a fluid area (or volume in 3-D), these bands exhibit a net degree of orientation that is identical to the time-averaged steady-state solution for the Couette flow.

In some flows, some or all of the fluid at all times is old fluid (e.g. a deflating magma reservoir). For such flows, the initial crystal orientation PDF is of fundamental importance in determining whether the PDF is periodic in time. The most reasonable initial condition is a perfectly random state given by a uniform PDF of crystal orientations. The PDF at any given location may then vary, perhaps periodically, as a function time. Nonetheless, the PDF at any moment in time is uniquely determined by the total strain, and hence by the specified boundary configuration.

Whereas models of crystal rotations based on Jeffery's (1922) solution typically have crystals with identical shape and identical aspect ratio, natural systems tend to be characterized by imperfect crystals of slightly differing shapes and aspect ratios. Furthermore, even in a dilute suspension of crystals, there will be occasional interactions between neighbouring crystals which lead to perturbations from Jeffery's solution. Here we provide a brief analysis of a system with a range of crystal aspect ratios in order to get an estimate of the effect on the orientation PDF and its time-periodicity. We choose a Couette flow geometry with crystal inclusions given by two different ranges of aspect ratios. For the first model, we use aspect ratios ranging from 2.5 to 3.5 , while for the second model, we use a range of 2.0-4.0. Both ranges are thus centred about an aspect ratio of 3.0. As our model only calculates PDFs for a single aspect ratio, we run the model a large number of times $(n=1000$; greater values of $n$ result in negligible differences in the results), each time for a slightly different aspect ratio. In the end, we add and normalize all resulting PDFs thus assuming that the aspect ratios for the two models are uniformly distributed over the given range. In Fig. 1, the results in terms of the degree of orientation $R$ are compared to the reference case with a single aspect ratio $a / b=$ 


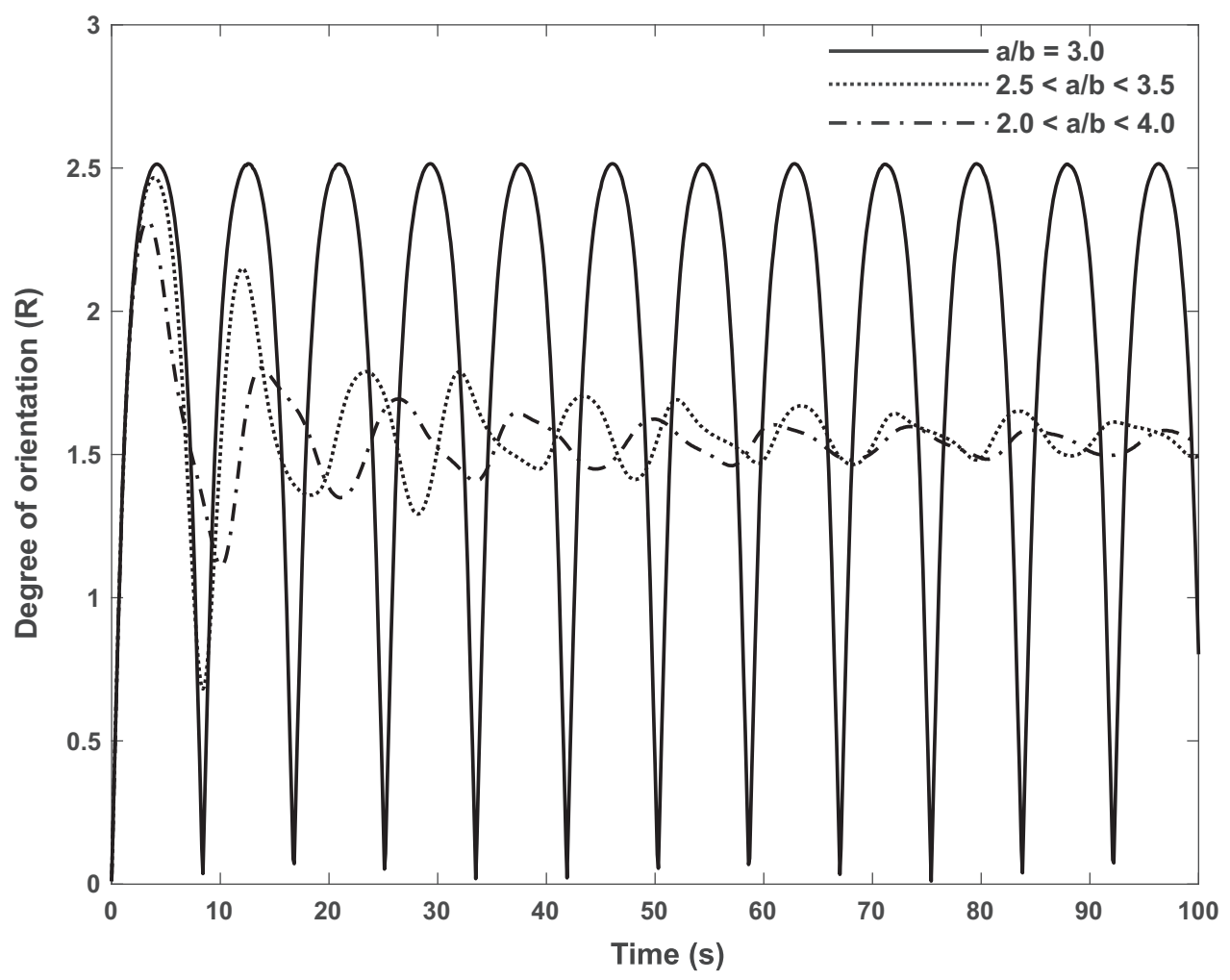

Figure 1. Evolution of the degree of orientation $R$ (see eq. 8) in a Couette flow for different assumptions of crystal aspect ratio $a / b$. The reference case with crystals of identical aspect ratio of $a / b=3$ illustrates the periodic behaviour of the PDF based on Jeffery's 1922 solution. The other two evolution curves are computed for uniformly distributed ranges of aspect ratios (see text for details). For distributed aspect ratios, the oscillatory behaviour is damped. The damping is greater for a broader range in aspect ratios. A full rotation of an individual crystal corresponds to two peaks of the evolution curve. For the two models with distributed aspect ratios, the periodic behaviour essentially vanishes after 4-5 crystal rotations, and the final degree of orientation of $R \approx 1.57$ is closely approximated by the time-integrated value calculated from eq. (23) in Bazargan et al. (2019).

3.0. Whereas the reference case is periodic for all time, the models with variable aspect ratios show a rapid decrease in the oscillation amplitude. After two crystal rotations (corresponding to four peaks in the reference curve), the amplitude of $R$ has decreased by a factor of 5 for the narrower range of aspect ratios, and by a factor of about 8 for the broader range. In both cases, the value of $R$ tends towards an asymptotic value close to the value of $R=1.57$ computed from eq. 23 in Bazargan et al. (2019). In nature, time-periodic behaviour of crystal rotations may thus be much less pronounced than predicted from the direct use of Jeffery's equations for a single crystal aspect ratio.

\subsection{General remarks about the 2-D PDF results}

For all models, we compute crystal orientation PDFs for elliptical crystals with a constant aspect ratio of $a / b=2$. Natural crystals often have a significantly higher aspect ratio, but we choose a relatively low value in order to generate conservative estimates for our results.

Throughout, we assume that the fluid has a constant viscosity. In magmatic systems, this is generally not the case, as the melt itself is governed by a temperature-dependent viscosity, and also the melt-crystal suspension has an effective viscosity which depends on crystal concentration and hence on temperature as well as composition (Giordano et al. 2008; Costa et al. 2009). The choice of using constant viscosity was made to keep the models simple, and to avoid a proliferation of results for different flow velocities and different temperature-viscosity functions. Geologically, our results may be interpreted as magmatic systems which developed rapidly (in comparison with the thermal diffusion timescale), and which ceased to flow because of a drop in the driving pressure, rather than progressive solidification of the magma.

For all flows, we show the velocity field because it is ultimately the source of all aspects of the crystal rotation dynamics, that is, the solid-body rotation rate, the pure shear stretching rate, and the advective velocity. Additionally, we show the vorticity number together with the direction of the stretching rate $\dot{\varepsilon}_{1}$ at each location because one may expect that the quantities have significant predictive power for the crystal PDFs. Lastly, we show maps of the most likely crystal orientation (i.e. the peak of the PDF) together with the degree of orientation as a summary of the complete 3D PDF field.

\subsection{Flow exiting or entering a planar channel}

For flow from a narrow planar channel into a significantly wider channel, the flow pattern near the outflow is similar to an outflow into an infinite reservoir. The velocity field is shown in Fig. 2(a). At the inlet into the channel, the velocity is specified as a constant value of $v_{x}=10 \mathrm{~m} \mathrm{~s}^{-1}$. Over a distance of approximately one channel width, friction against the channel walls causes a Poiseuille flow to develop with a maximum velocity of $v_{x}=15 \mathrm{~m} \mathrm{~s}^{-1}$ at its centre (cf. Turcotte \& Schubert 2014). Near the transition from channel to reservoir, the velocity in the channel gradually decreases because of the increase in channel width due to the rounded corners of the transition. Within the reservoir, the flow spreads out approximately 
(a)

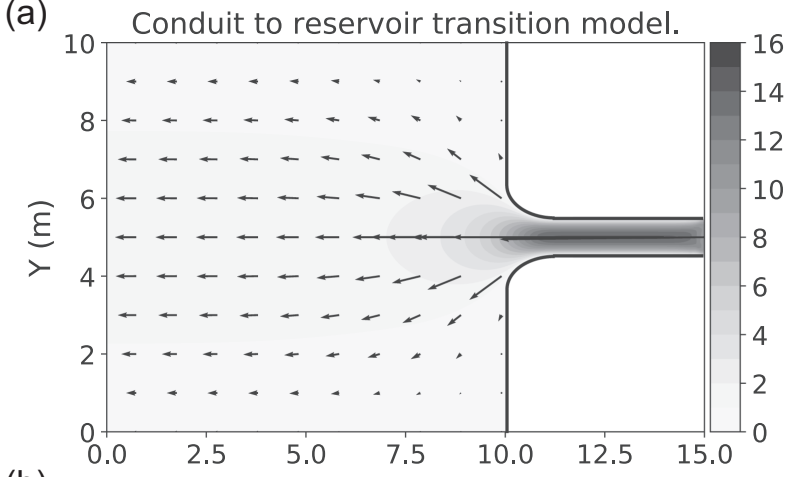

(b)

(c)
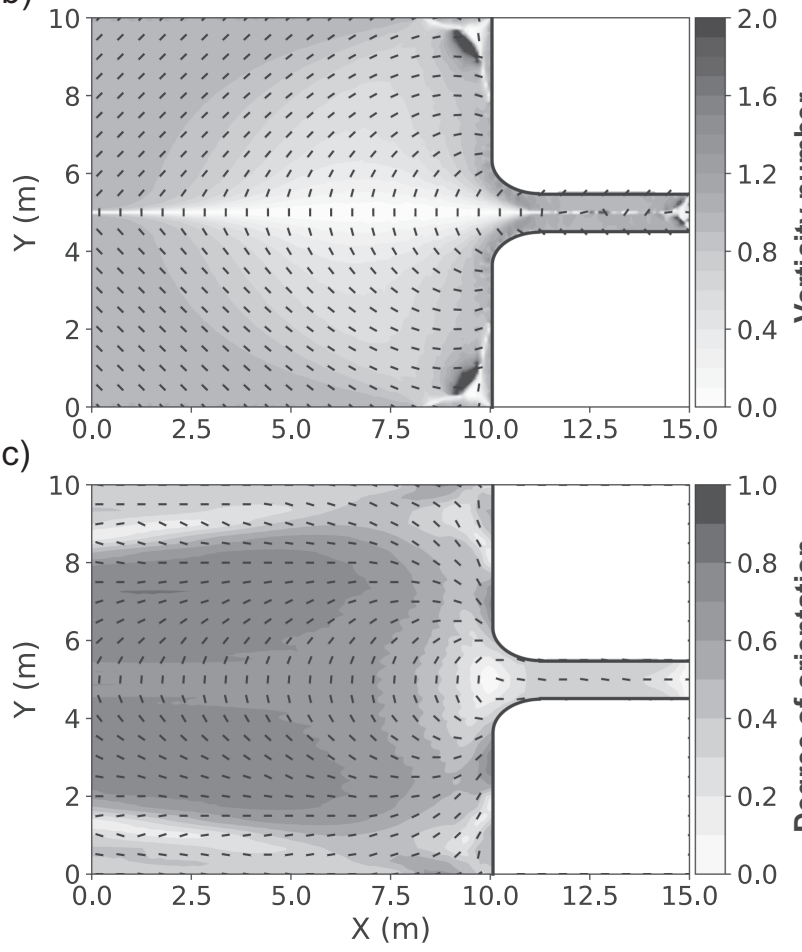

(d)

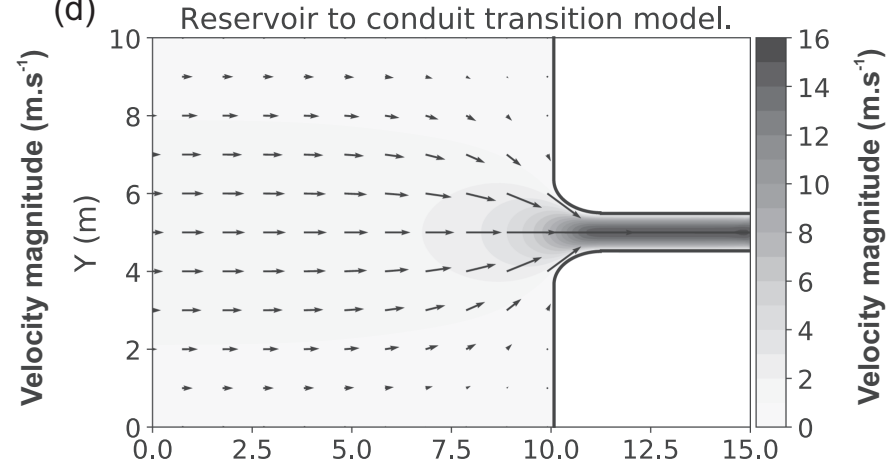

(e)

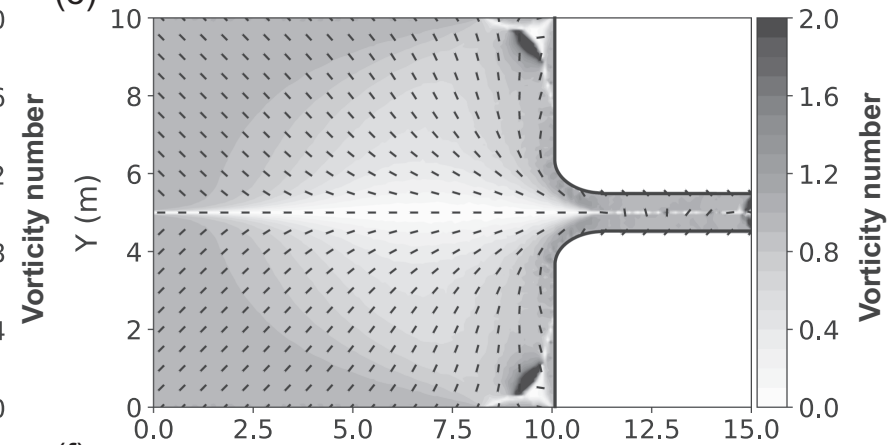

(f)

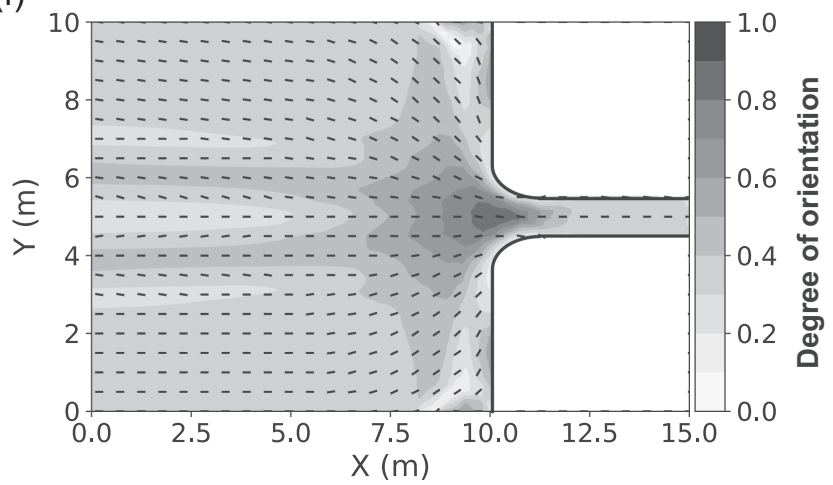

Figure 2. Results for the models of the transition between channel and reservoir. Left-hand panels are for flow exiting from the channel into the reservoir, right-hand panels are for the reverse flow. (a) and (d) Velocity magnitude (shading) and direction (arrows) of the fluid flow. (b) and (e) Vorticity number (shading) and orientation of $\dot{\varepsilon}_{1}$ (tick marks). (c) and (f) Degree of orientation of the PDF (shading) and most likely orientation (peak of PDF) of particles with aspect ratio $a / b=2$ (tick marks). In the conduit-to-reservoir model, particles mostly align perpendicular to the flow direction, while in the reservoir-to-conduit model, particles align in the direction of flow.

radially from the centre of the outflow, and the magnitude of the velocity decreases accordingly with approximately $1 / D^{2}$, where $D$ is the distance from the channel exit. At large distances from the channel exit, the geometry of the reservoir affects the velocity field. Far downstream from the narrow inlet, the larger reservoir effectively acts as a much larger channel, and a parabolic velocity profile typical of a Poiseuille flow develops over a distance of about one channel width (i.e. about $10 \mathrm{~m}$ ).

The pattern of pure-shear stretching (orientation of the tick marks in Fig. 2b) clearly shows the $45^{\circ}$-alignment of the $\dot{\varepsilon}_{1}$-axis in the inlet channel, which is characteristic of Poiseuille flow. At the centre of the channel, the strain rates are zero, and the observed erratic orientations of $\dot{\varepsilon}_{1}$ there are due to numerical noise. At the channel exit, as the flow spreads out radially, the fluid contracts in the direction of the flow, and hence (by conservation of mass) extends in the perpendicular direction. The vorticity number is near unity throughout much of the domain, with small regions of elevated $W_{k}$ near the corners of the reservoir and at the stress-free outflow from the modelled domain.

The resulting crystal orientations, summarized in Fig. 2(c), show rather complex behaviour. In the Poiseuille flow of the inlet channel, the crystals rapidly orient themselves parallel to the flow direction. The detailed behaviour is much more complex than what the spatial resolution allows the model to show. As illustrated in a previous analysis of a Poiseuille flow with a uniform PDF at the inlet, the crystal orientations develop into bands of spatially constant PDF which are nearly parallel to the flow (Bazargan et al. 2019). The width of the bands decreases towards the edge of the channel, with infinitesimally narrow bands at the channel walls. As the uniform PDF at the inlet and as initial condition does not lead to a stationary solution, the bands continually evolve. The time-averaged behaviour is a preferred crystal orientation that is exactly parallel to the flow, and a degree of orientation of $R \approx 0.3$. This averaged behaviour is well captured by the model. Past the channel 
exit, the flow regime changes. As a result, the crystals have to realign, and because of this, there is a significant decrease in $R$ along the flow axis.Beyond a distance of about two widths of the narrow channel (i.e. about $2 \mathrm{~m}$ ) downstream of the exit, strong crystal alignment develops due to the flow-perpendicular pure stretching. The peak of the PDF of orientations in this regime is perpendicular to the flow direction, and a very high degree of orientation develops with values approaching $R=0.8$. Near the reservoir walls, the flow is parallel to the boundary, and within the frictional boundary layer, the flow is well approximated by a Couette flow. In this region, the preferred crystal orientation is thus approximately parallel to the wall. The degree of orientation tends to be lower than in the centre of the flow, with values around $R \approx 0.3$, but with significant variation due to advection of the previously developed pattern.

We inverted the flow direction of the model by changing the outflow to an inflow with constant normal velocity, and at the same time changing the inflow to a stress-free outflow. The model then simulates the flow around the entrance into a planar channel. Due to our choice of boundary conditions, the velocities near the inand outflow boundaries are not exactly equal to the inverse of the velocities of the previous model. At the centre of the model domain, however, these differences vanish, and the velocity field is effictively equal in magnitude and opposite in direction compared to that of the channel exit (Fig. 2d). Since the flow field is incompressible, the condition $\dot{\varepsilon}_{1}=-\dot{\varepsilon}_{2}$ holds everywhere, and hence the principal strain rates of the channel entrance model are obtained by exchanging $\dot{\varepsilon}_{1}$ and $\dot{\varepsilon}_{2}$ (Fig. 2e). The degree of crystal orientation is significantly lower throughout most of the model domain compared with the model of the channel exit (Fig. 2f). The reason for this is mostly the advection direction. In the channel entrance model, unaligned crystals enter from the left-hand side of the domain. The strain rates are low throughout the central part of the broad channel, and hence the crystals travel a long distance before any orientation fabric can develop. Near the channel walls, the flow is largely characterized by simple shear, a conclusion supported by the fact that the degree of orientation increases to $R \approx 0.3$, and then stagnates around that value. As the fluid is drawn in a radial direction into the entrance of the narrow channel, two lobes of high $R$ develop, oriented about $45^{\circ}$ relative to the axis of the channel. The maximum value of $R \approx 0.8$ occurs just past the entrance into the narrow channel. Beyond this point, a Poiseuille flow develops with flow-parallel crystal orientations and $R=0.3$.

\subsection{Inflating and deflating reservoirs}

In the model of the inflating magma reservoir, the velocities are approximately radially outward from the inflow, and the velocity magnitude decreases away from the inlet at the base (Fig. 3a). Since the boundary of the reservoir expands in time, the normal fluid velocity at this boundary is not equal to zero in absolute terms (although it is zero relative to the moving boundary). The vorticity number is close to zero along the vertical axis of the reservoir, and approaches unity near the reservoir walls in the lower half of the domain (Fig. 3b). The flow field is dominated by pure shear stretching parallel to the reservoir walls, and contraction in the perpendicular direction. However, the flow inlet perturbs the flow pattern in the lower half of the reservoir, causing the stretching axis $\dot{\varepsilon}_{1}$ there to be inclined by about $45^{\circ}$ relative to the walls. High values of solid body rotation are limited to the immediate vicinity of the entrance of the reservoir. With relatively low solid body rotation rates and high pure shear rates, strong preferred crystal fabrics develop parallel to the walls at the edges of the reservoir, and perpendicular to the flow direction near the centre. The maximum value of $R$ occurs in the top third of the reservoir along the flow axis, with a value of $R \approx 0.9$. Interestingly, the degree of orientation is lower along the boundaries, with $R \approx 0.4-0.5$. This effect can be explained by a simple thought experiment. If the chamber inflation were accommodated by uniform (e.g. elastic) stretching of the material inside, then all parts of the reservoir would experience the same strains and hence the same crystal rotations. For the incompressible fluid of our experiment, the strain rates along the reservoir edges are the same as for the uniform stretching case (i.e. the total strain along the edge is dictated by the boundary condition). Fluid closer to the centre experiences the same pure shear strain as part of the chamber inflation, but due to the incompressibility condition, this part of the fluid simultaneously has to move closer to the chamber wall. This radial motion causes additional stretching parallel to the wall, and compression perpendicular to it. As a result, the fluid that starts out close to the centre of the reservoir and then moves outward develops the strongest degree of crytal orientation. Near the inlet, the flow along the walls is dominated by simple shear flow, and the degree of orientation remains close to $0.3-0.4$.

The deflating reservoir is characterized by velocities in the direction opposite those of the inflation model (Fig. 3d), and the corresponding orientations of $\dot{\varepsilon}_{1}$ are perpendicular (Fig. 3e). As a result, the preferred crystal orientations are more or less aligned with the flow direction (Fig. 3f). The degree of orientation along the top half of the boundary is similar to that of the inflation model, thus lending support to the thought experiment about uniform stretching in a circular reservoir. The degree of orientation increases along the flow paths because this material has experienced additional stretching in the direction of the flow due to the motion from a larger radius towards a smaller radius within the reservoir. The highest degree of orientation of $R \approx 0.8$ is attained just before the fluid exits the reservoir.

\subsection{Magma flow in channels with sharp bends}

In the straight parts of a planar channel, the flow is observed to be a typical Poiseuille flow (Turcotte \& Schubert 2014) with parabolic velocity profile. Near a bend, the flow pattern is perturbed over a distance of approximately one channel width up- and downstream from the centre of the bend. This observation holds for the $45^{\circ}$ and for the $90^{\circ}$ bend as well as for the T-shaped junction. Because of the similarity of the results of the two kinked channels, we show only the results for the $45^{\circ}$ bend (Fig. 4). The velocity pattern through the bend itself is similar to the Poiseuille flow, but the location of maximum velocity is shifted towards the inside corner as the flow follows a smoother, curved path relative to the sharp turn of the conduit. Both the velocity field (Fig. 4a) and the vorticity number (Fig. 4b) show that the flow pattern is symmetric about the axis of the bend. This symmetry is broken in the PDF field (Fig. 4c) because of the effect of advection of the crystal orientations. This asymmetry is clearly seen in the intensity pattern of the crystal orientations, which exhibits a maximum with a magnitude of about $P=0.5$ located downstream and towards the outer edge of the bend. The dominant orientation of the crystals, however, is essentially unaffected by the bend, and more importantly, no asymmetry about the axis of the bend can be discerned (see tickmarks in Fig. 4c). This means that it is probably impossible to determine the magma 
(a)

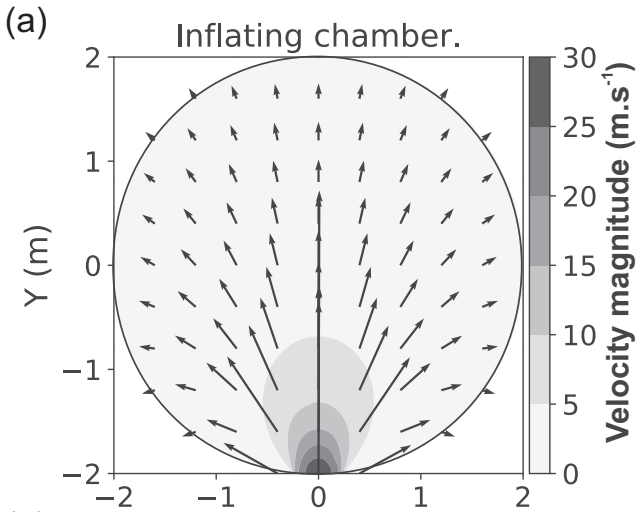

(b)

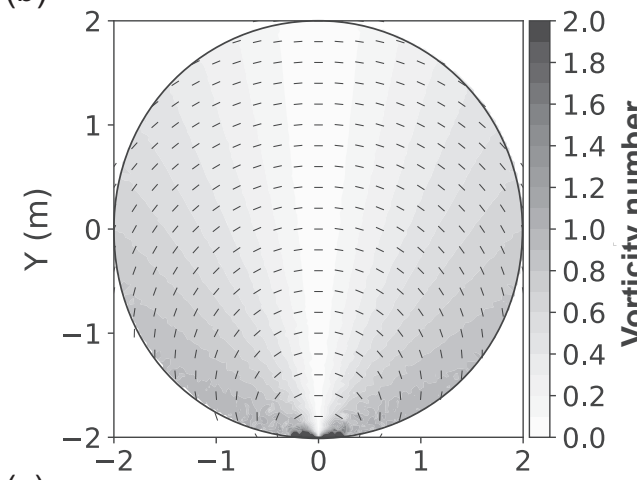

(c)

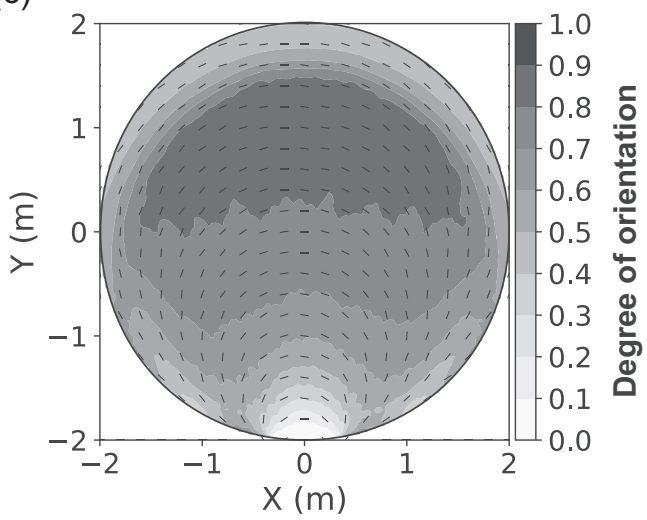

(d)

(e)
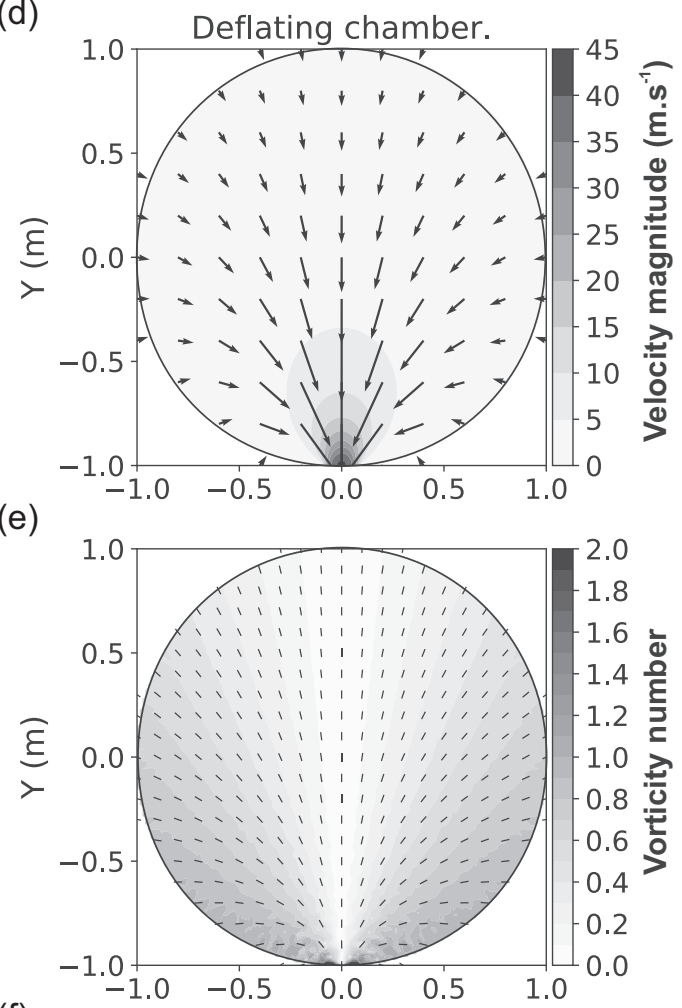

(f)

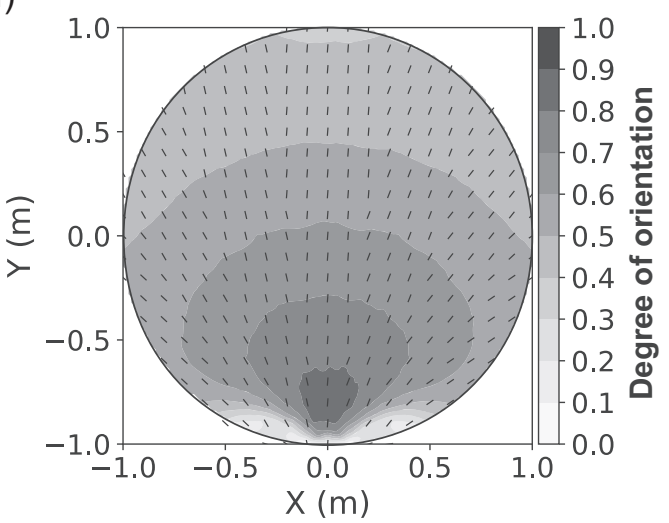

Figure 3. Results for the models of inflation and deflation of a magma reservoir. Fluid enters or exits the model domain via an inlet or outlet located at the bottom of the reservoir. (a) and (d) Velocity magnitude (shading) and direction (arrows) of the fluid flow. (b) and (e) Vorticity number (shading) and orientation of $\dot{\varepsilon}_{1}$ (tick marks). (c) and (f) Degree of orientation of the PDF (shading) and most likely orientation (peak of PDF) of particles with aspect ratio $a / b=2$ (tick marks). In the inflation model, particles mostly align perpendicular to the flow, while in the deflation model, particles align with the flow.

flow direction in a dyke from observations of crystal orientations near a bend or kink in the dyke.

The flow from a vertical dyke via a T-junction into a horizontal sill is similar to the flow in a kinked dyke in that high-velocity zones are channeled towards the inside of the turn, whereas flow in the outside curve (i.e. in centre of the dyke-sill transition) displays very low velocities (Fig. 5a). The vorticity number is high within the high-velocity zones at the edge of the junction, while it is low (near zero) in the stagnation zone at the centre of the junction (Fig. 5b). The crystal orientations (Fig. 5c) are mostly aligned with the flow direction, except in the stagnation zone in the upper part of the junction. In the stagnation zone, the solid body rotation rate of the fluid is nearly zero (i.e. zero vorticity number), and hence the crystals align with the $\dot{\varepsilon}_{1}$-axis, which is horizontal. The degree of orientation is near unity (i.e. perfect alignment with the PDF approaching a Dirac delta function) in a small region at the top of the junction.

\subsection{Thermal convection in magma reservoir}

Due to the low Rayleigh number of the flow $(R a=1400)$, a stable convection pattern develops with two upwellings and two downwellings (Fig. 6a). The strain rate field is dominated by the source zones of the up- and downwellings where $\dot{\varepsilon}_{1}$ is vertical, and by the boundary regions with impinging up- and downwellings, where $\dot{\varepsilon}_{1}$ is horizontal and thus parallel to the boundary (Fig. 6b). Along the boundaries, the flow between the up- and downwellings is essentially a Couette flow, with $W_{k} \approx 1$, and with $\dot{\varepsilon}_{1}$ oriented at $45^{\circ}$ relative to the boundary. High values of the vorticity number of around $W_{k}=10$ are found in small zones to the left and to the right 
(a)

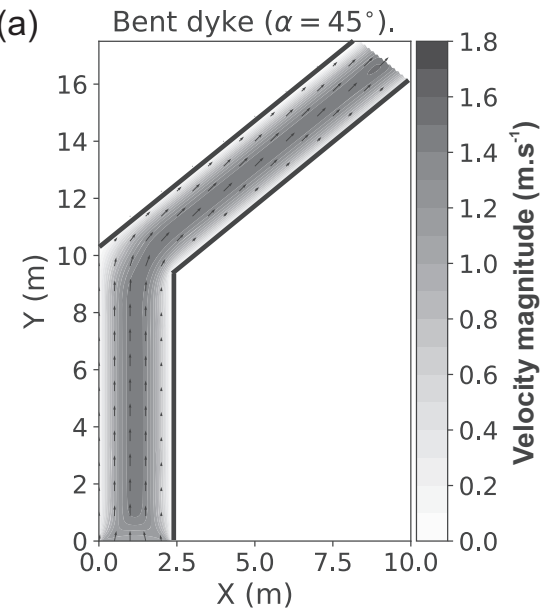

(b)

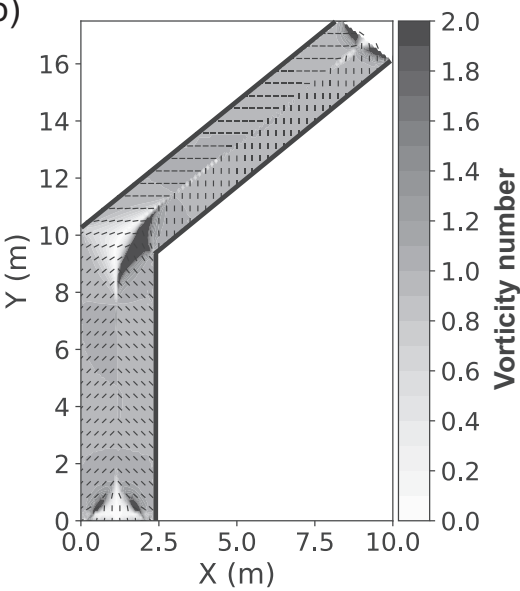

(c)

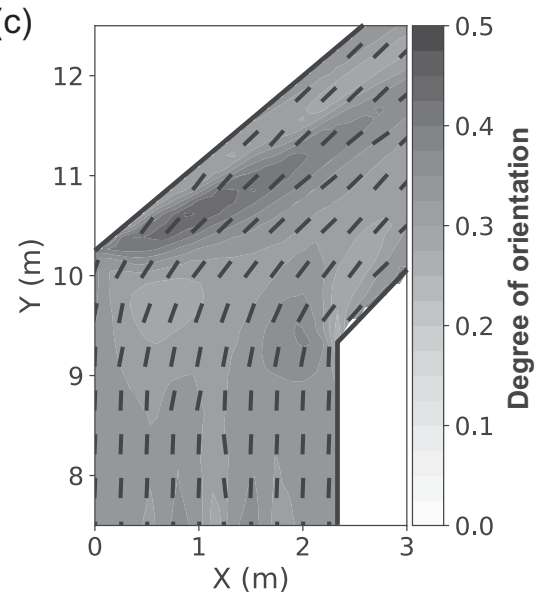

Figure 4. Flow of magma through a planar channel with a sharp $45^{\circ}$-bend. (a) Velocity magnitude (shading) and direction (arrows). (b) Vorticity number (shading) and orientation of $\dot{\varepsilon}_{1}$ (tick marks). (c) Degree of orientation of PDF (shading) and most likely orientation (peak of PDF) of particles with aspect ratio $a / b=2$ (tick marks). The flow is symmetric about the centre of the bend. The resulting pattern of most likely crystal orientations is also approximately symmetric, while the degree of orientation has a maximum downstream from the bend. With present analysis techniques of field data, it is probably not possible to determine the flow direction from field observations of a kinked dyke. For a sharp $90^{\circ}$-bend, the results are qualitatively the same and are therefore not shown here. of the centre of the convection cells. Due to horizontal heat conduction, the convection cells are not perfectly symmetric about the horizontal axis of the domain. As the cell spins around its centre, it stretches more towards the horizontally convergent portions of the up- and downwellings than it does towards the horizontally divergent ends. This asymmetry of the convection cells causes the peaks of the vorticity number field to be shifted relative to the horizontal centre line. At the base of the up- and downwellings, the vorticity number is low, and is identically equal to zero at the stagnation point.

The field of crystal orientations can be described in terms of three different zones. Starting at the base of the up- and downwellings, a vertical PDF pattern develops that increases in strength until the flow has passed the mid-elevation of the domain. Beyond this point, the degree of orientation decreases again. The width of the vertically oriented zone increases along the flow direction. Away from the base of the vertical flows, a second type of zone can be distinguished along the reservoir walls. The PDF along the boundaries is characterized by horizontal crystal orientations. The degree of orientation decreases in the flow direction from $R \approx 0.7$ near the impinging plume to $R \approx 0.3$ just before the flow enters the next plume. The centres of the convection cells comprise the third regime of crystal orientations. Here, the orientations are generally subhorizontal, and aligned at about $45^{\circ}$ relative to the local orientation of the $\dot{\varepsilon}_{1}$-axis. The degree of orientation in the middle of the convection cells is low, with typical values of $R \approx 0.1-0.2$, but with irregular zones of higher values up to $R \approx 0.5$. The observed preferred crystal orientations are observed to be constant in time. The centres of the convection cells thus do not merely spin about their centre, as such a rotation would lead to secular changes in the orientation field. Rather, the flow continuously stretches and deforms because of the quadrilateral shape of the cells and because of their aspect ratio, which is different from unity. At the transition between convection cells and boundary layer, as well as at the transition between convection cell interior and plumes, there is a narrow belt of low degree of orientation $(R \approx 0-0.1)$ and with fairly random dominant crystal orientations.

Since the convective flow is in steady state with spatially fixed up- and downwellings, it is impossible from the results shown to estimate how rapidly features are created or destroyed in the crystal orientation PDF. However, for a correct interpretation of the magma dynamics, it may be critical to know whether the observed PDF represents an instantaneous snapshot of the flow conditions, or instead is the result of a time-integration of flow conditions that persisted for a long duration. In order to shed light on this question, we ran the same convection model with a checkerboard pattern in $x-y$-space for the initial condition of the crystal orientation PDF. The size of each checkerboard square is $10 \mathrm{~m}$ by $10 \mathrm{~m}$. Alternating squares are given preferred crystal orientations of 0 and $\pi$ with a width of two elements for the PDF peak in $\theta$. Properly normalized, the PDF is thus set to a constant value of 5.093 over a $\theta$-range of 0.196 radians. The results (Fig. 7) show that the convective flow largely destroys a given pattern of the crystal orientation PDF over a timescale that corresponds to the time taken by a fluid parcel located in a focused up- or downwelling to traverse the height of the convective domain. Given the flow pattern, this timescale corresponds to approximately 20 per cent of a complete convective overturn. After a time of around 10 per cent of a convective overturn, the original pattern is strongly distorted, and the peak values of the PDF have decreased from their original values of $P=$ 5.093 to around $P \approx 2-3$ near the boundaries and to $P \approx 0-2$ 
(a)

Dyke to sill.

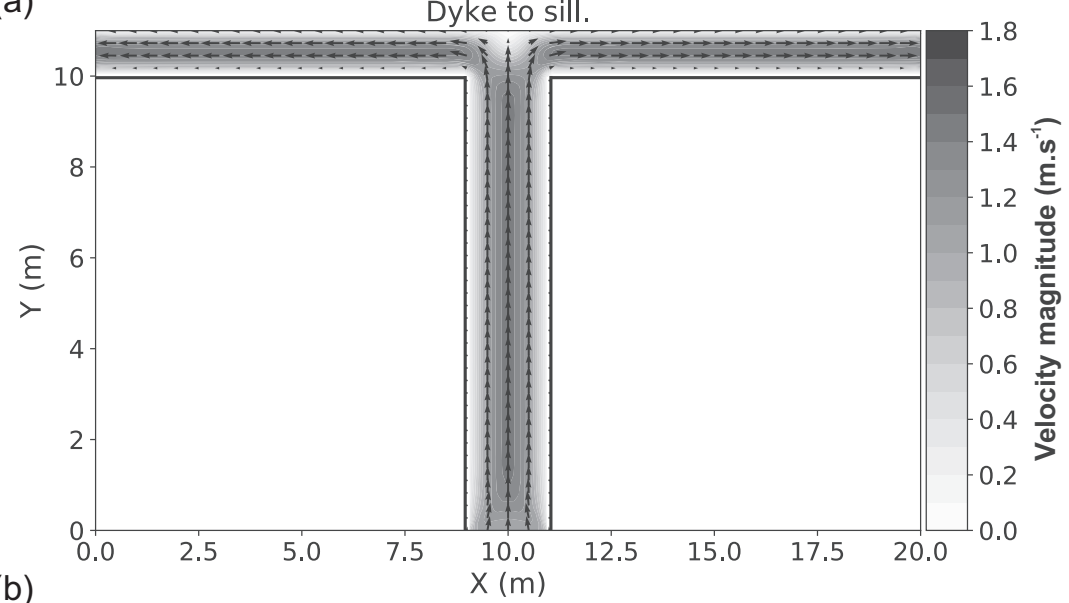

(b)
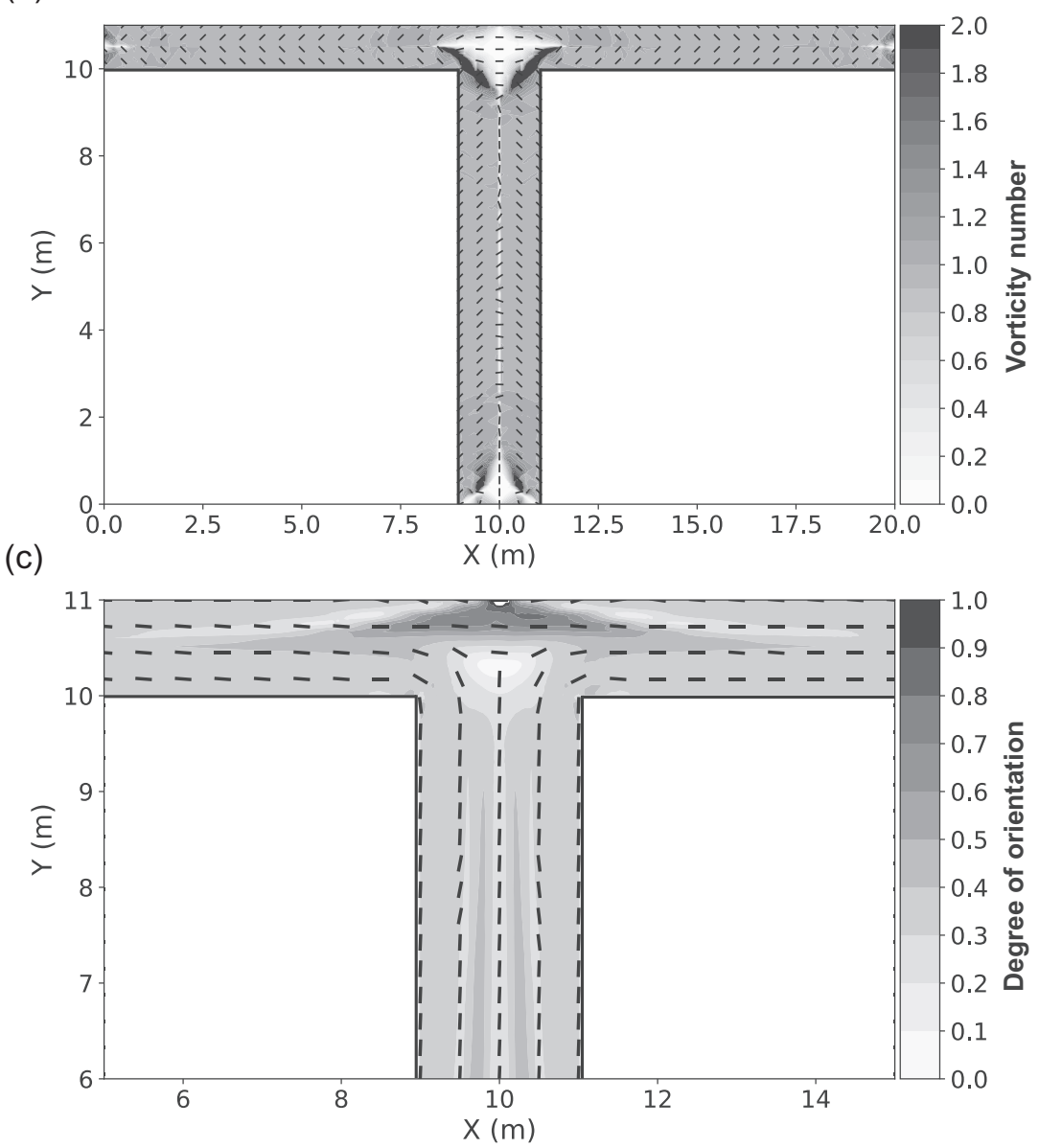

Figure 5. Magma flow from a vertical dyke into a horizontal sill. (a) Velocity magnitude (shading) and direction (arrows). (b) Vorticity number (shading) and orientation of $\dot{\varepsilon}_{1}$ (tick marks). (c) Degree of orientation of PDF (shading) and most likely orientation (peak of PDF) of particles with aspect ratio $a / b=2$ (tick marks). Most of the domain is governed by plane Poiseuille flow with corresponding flow-parallel crystal orientations. Within the transition, the crystals rapidly take an orientation parallel to the horizontal sill. The orientation change inside the junction is due to pure shear deformation similar to the outflow from a channel into a reservoir. At the top centre of the junction, there is a stagnation point where the velocity approaches zero and the degree of crystal orientation approaches unity (i.e. a Dirac delta function in the PDF).

at the centre of the flow. After a time of 20 per cent of a convective overturn, the original checkerboard pattern is so strongly distorted that it can no longer be discerned. However, the PDF has not been homogenized completely, and remnants of the original pattern still persist with PDF peaks of magnitude $P \approx 2$. Destruction of the original pattern and formation of the new, steady-state pattern is essentially complete after a time corresponding to one to two complete overturns. 
(a)

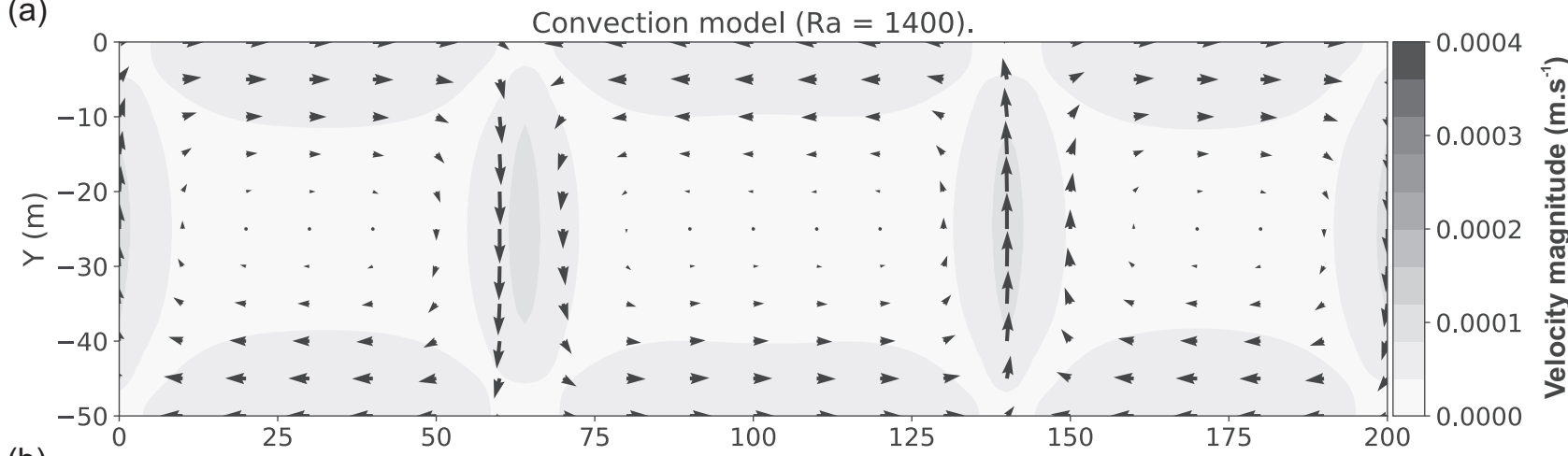

(b)
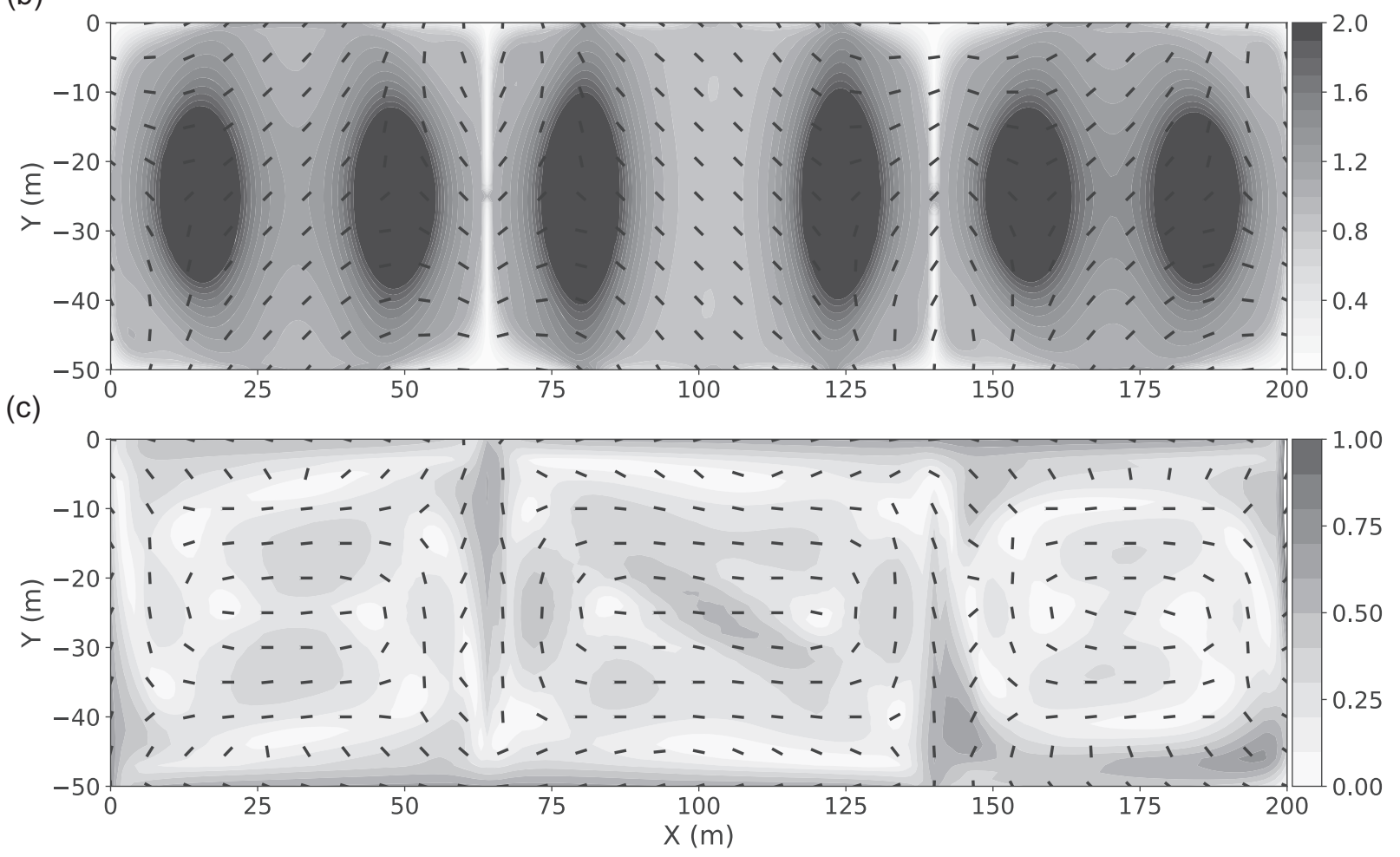

Figure 6. Results for thermal convection with Rayleigh number of 1400. (a) Velocity magnitude (shading) and direction (arrows) of the fluid flow. (b) Vorticity number (shading) and orientation of $\dot{\varepsilon}_{1}$ (tick marks). (c) Degree of orientation of the PDF (shading) and most likely orientation (peak of PDF) of particles with aspect ratio $a / b=2$ (tick marks).

\subsection{Effect of crystal aspect ratio}

Throughout our results, we assumed a constant crystal aspect ratio of $a / b=2.0$. In this section, we show results for different aspect ratios with values $a / b=4.0$ and $a / b=8.0$. We limit our discussion to the results for the flow geometry of the channel-to-reservoir transition because this model locally incorporates different flow regimes such as plane Poiseuille flow in the inlet channel and divergent flow within the transition. The most striking difference between identical models with different crystal aspect ratios is that the range of the degree of orientation is greater for larger aspect ratios. Whereas the minimum degree of orientation at the centre of the transition remains relatively constant with values near zero, the maximum degree of orientation is about $R=0.7$ for a crystal aspect ratio of $a / b=2.0$ (Fig. 2c), and it is about $R=0.93$ for $a / b=4.0$ and about $R=$ 0.97 for $a / b=8.0$ (Figs $8 \mathrm{a}$ and $b$ ). The shape of the contours of the degree of orientation is similar for the three different crystal aspect ratios, but they are not identical. Perhaps most notably, for greater aspect ratios, the maximum of the degree of orientation $R$ is shifted further upstream. The reason for this is that more elongate crystals rotate more rapidly when they are approximately perpendicular to the local alignment direction, while they rotate more slowly when they are close to local alignment (Jeffery 1922; Bazargan et al. 2019). Taken together, these rotation effects cause elongate crystals to reach local alignment more rapidly. Since the flow and thus the advection velocity are the same for all models, maximum alignment in a locally coherent flow is reached further upstream for more elongate crystals.

The general conclusions of this section also hold for the other flow geometries. The crystal alignment patterns shown for an aspect ratio of $a / b=2.0$ are thus geometrically similar to the patterns for other aspect ratios, but the degree of orientation is greater for greater aspect ratios. 
(a)

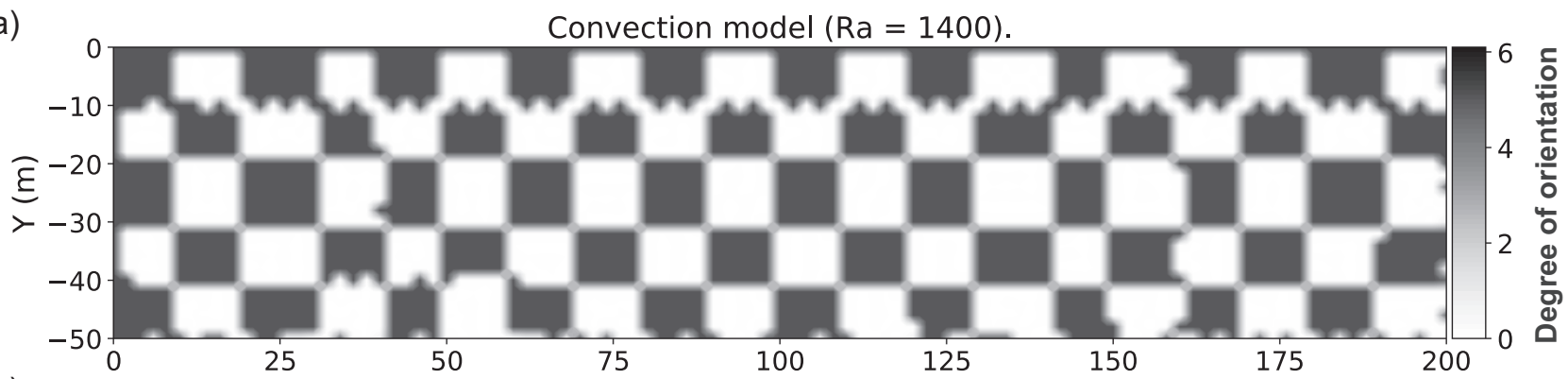

(b)

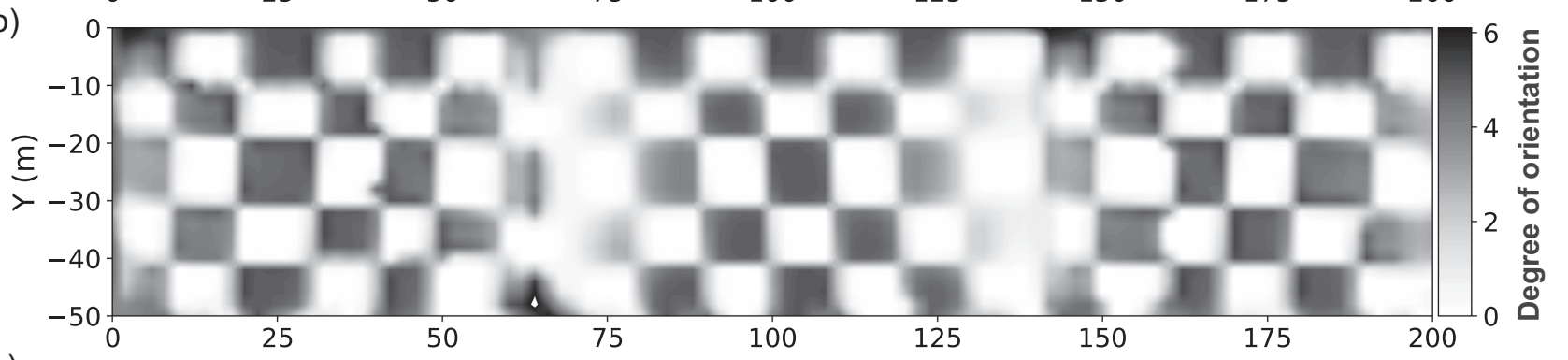

(c)

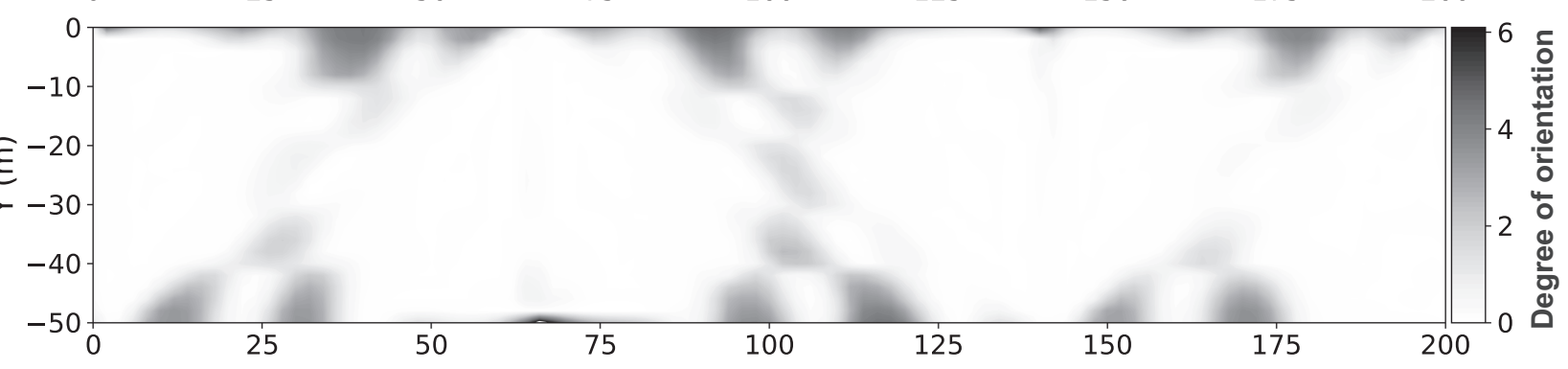

(d)

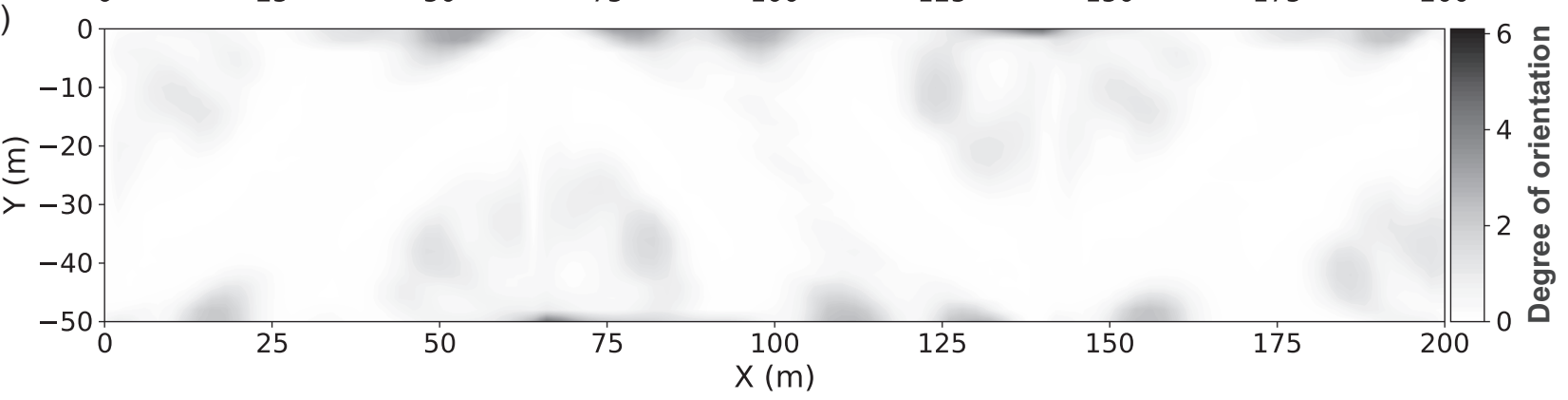

Figure 7. Values of the PDF of crystal orientations as a function of $x$ and $y$ for a slice in orientation-space taken at $\theta=0$. (a) The original checkerboard pattern $(t=0)$ shows alternating squares of strong alignment $(P=5.093)$ and no alignment $(P=0)$ about the value of $\theta=0$. In other words, the squares with high values of $P$ have approximately horizontal crystal orientations, while the squares with low values of $P$ have approximately vertical crystal orientations. (b) After a time of $t=2 \times 10^{4} \mathrm{~s}$ ( $\mathrm{ca} .0 .7$ per cent of a convective overturn), the pattern is largely unchanged, but the magnitude of the peaks has decreased in the high-velocity zones of the up- and downwellings. (c) At time $t=2.6 \times 10^{5} \mathrm{~s}$ ( $\mathrm{ca} .9 .5$ per cent of an overturn), the pattern has largely been destroyed in the high-velocity zones, while a distorted pattern is still discernible along the horizontal boundaries and at the centre of the convection cells. (d) After a time of $t=5.5 \times 10^{5} \mathrm{~s}$ ( ca. 20 per cent of an overturn), the details of the pattern have disappeared entirely, but some variability remains in the PDF field as a result of the initial conditions.

\section{DISCUSSION}

\subsection{Comparison with analog models and with field observations}

The model results of this study show how a number of relatively simple, 2-D flows lead to a great variety of crystal orientation patterns with variable strength of the orientation fabric. Analog models provide a set of rigorous tests for our modelling approach because their velocity fields are generally well constrained. Overall, we find good agreement between our theoretical calculations and the observed alignment fabric in analog experiments.
For example, Trebbin et al. (2013) analysed the alignment of deformable polymeric micelles in laminar flow of water from a wide channel into a narrower section and back into a wider channel. The aspect ratio (i.e. length to diameter) of the micelles is around 100, which is much greater than the aspect ratio of 2 which we used in our experiments, and their transition between the two channel widths is significantly longer than in our model. Nonetheless, the overall alignment pattern of their micelles corresponds very closely to our models of flow from a channel into a reservoir and vice versa (Fig. 2). The similarities are most striking in the channel expansion, where the alignment of inclusions changes from flow-parallel to 
(a)

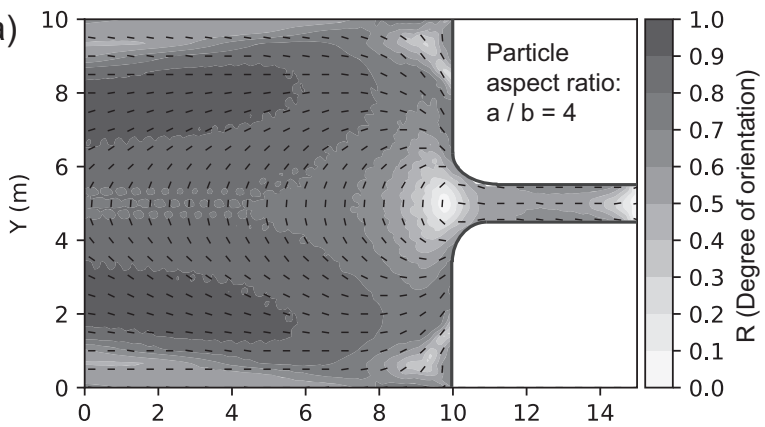

(b)

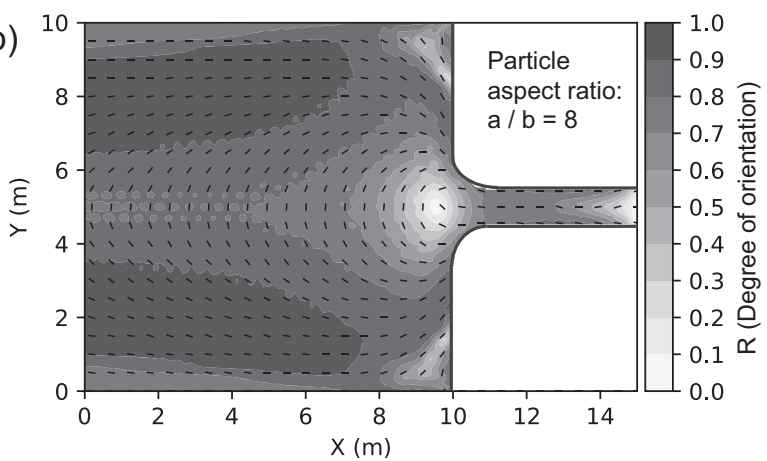

Figure 8. Model results for the transition from channel to reservoir illustrating the effect of varying crystal aspect ratio. Degree of orientation $R$ of the PDF (shading) and most likely orientation (peak of PDF) for particles with aspect ratio (a) $a / b=4$ and (b) $a / b=8$. Results for the same flow model with crystal aspect ratio $a / b=2$ are shown in Fig. 2(c). The crystal alignment patterns are geometrically similar but not identical. The degree of orientation is greater for higher aspect ratios. We find that these qualitative results also hold for other model geometries (not shown).

flow-perpendicular. The alignment intensity is greatest at the centre of the channel after the flow has passed the expansion, whereas the flow near the walls is everywhere dominated by simple shear flow and a corresponding intermediate degree of orientation. At the inflow to the channel contraction, the degree of orientation in their experiment shows a very weak minimum in approximately the same position where our model predicts a relatively strong minimum. As their channel transition (from wide to narrow) extends over a distance greater than 10 times the thickness of the narrow section, the flow in the contraction probably remains closer to a Poiseuille flow throughout, and the patterns observed in our numerical experiments are expressed with much lower intensity. The fundamental principle that describes the crystal alignment in inand outflows from a reservoir has also been described by Paterson et al. (1998). They introduce the terminology of 'convergent flow' for the transition from reservoir to channel, and 'divergent flow' for the transition from channel to reservoir. Based on a conceptual analysis of the flow, they qualitatively predict the alignment pattern displayed by our analytical results. A similar divergent flow pattern is encountered in models of magma entering a lava dome, using plaster of Paris (with a non-linear rheology) as an analog for the magma (Závada et al. 2009). Also in these models, elongate inclusions orient themselves perpendicular to the flow direction where the flow spreads out radially. For this type of flow condition, there is strong agreement between the analog models and our numerical results.

A flow with a large component of expansion against receding and stretching reservoir walls analogous to our model of an inflating magma chamber was studied in a model of diapiric ascent of plaster with embedded magnetite crystals into a host of fine-grained sand (Kratinová et al. 2006). Within the plume head, where the similarity between the two flows is greatest, the crystals are aligned with the walls of the intrusion. The degree of orientation in the diapir head is relatively large. Qualitatively, we find good agreement between our model and the observed crystal alignment in the diapir head. In the lower parts of the diapir, both the deformations and the crystal alignment indicate Poiseuille-type flow near the base and flow divergence near the centre of the diapir. In a different setup, Buisson \& Merle (2002) studied the intrusion of silicone putty into a pre-existing dome of the same material. The flow does not contain any inclusions, but instead the strain ellipses are directly visualized by means of an initial pattern consisting of a regular grid and circles which is printed in carbon onto one of the lateral sides of the dome. Since the orientation of the strain ellipsoid is identical with the direction of the peak of the orientation PDF for needle-like inclusions (March 1932), the experiment effectively provides the alignment pattern of inclusions with very high aspect ratio. With the flow approximately radially outward from the inflow, the strain ellipses are generally parallel to the outer boundaries of the dome. The deformation of the ellipses is greatest just outside the zone of newly intruded material. Both the alignment pattern and the degree of orientation are thus in good agreement with our numerical results.

Direct evidence of convection in a magma reservoir based on crystal orientations is scarce. One possible example is the study of the La Gloria pluton by Payacán et al. (2014) and by Gutiérrez et al. (2013). They construct a convection pattern on an exposed cross section of the pluton based on a number of observations of magnetic and magmatic fabrics. Our results lead to a slightly different interpretation of the flow field because a boundary-perpendicular crystal orientation appears to be a strong indication of the base of an up- or downwelling (Fig. 6c). Given the relatively small number of observation points in the study by Payacán et al. (2014), it is difficult to construct the complete convection pattern. The process is made more complicated by the third dimension, which is not accessible in the observed pluton, but which may have important effects on the flow pattern and the crystal rotations. Given the size of the pluton, dynamic considerations (i.e. estimates of the Rayleigh number) indicate that thermal convection is likely to have occurred in this magma body. With more data points, it may be possible to show unequivocally that the pattern of magnetic lineations was caused by convection.

\subsection{Determining the flow field from observations of crystal orientations}

Given the velocity field and its evolution in time, it is relatively straightforward to understand conceptually how the flow field causes the observed PDF field of crystal orientations. The inverse of this process is what is generally desired in field observations, but this approach is more difficult because the knowledge of the local crystal orientations does not allow a direct determination of the flow geometry or the flow direction. For example, net crystal alignment along a flow boundary often indicates boundary-parallel shear flow, as in Couette flow, in Poiseuille flow, and in the models of this study which simulate a transition between a narrow channel and a reservoir. However, boundary-parallel crystal alignment is also observed in the model of the inflating reservoir, and in the thermal convection model. In these two models, boundary-parallel alignment is due to pure shear deformation with negligible solid body rotation. 
Boundary-parallel fabric may thus indicate either simple shear flow along the boundary, or boundary-parallel pure shear stretching. Typically, the degree of orientation is greater for flows with pure shear because these flows are free from solid-body rotation. A more detailed analysis shows that the boundary-parallel fabric in simple shear flows is due to an orientation of the $\dot{\varepsilon}_{1}$-axis that is inclined by $45^{\circ}$ relative to the boundary coupled with a solid-body rotation (Bazargan et al. 2019), whereas the zone of an impinging upor downwelling is characterized by boundary parallel stretching, with the $\dot{\varepsilon}_{1}$-axis aligned with the wall. From the local picture of crystal orientations alone, it is impossible to distinguish these two regimes. Additional complications in interpreting observations of crystal orientations are due to advection of the PDF field. Except in the simplest of cases, it therefore seems likely that an accurate interpretation of a given field of crystal orientations requires computation of a forward model of the (assumed) complete flow field, coupled with the computations of the crystal rotations.

A fundamental issue that arises regards the uniqueness of the solution. It is clear that the forward model, for given initial and boundary conditions, always yields a unique solution for the crystal orientation field. But does a given crystal orientation field allow the unique determination of the fluid's velocity field? The answer is no, as it is straightforward to construct an example of two flow fields which result in the same crystal orientation field. Consider two uniform flow fields that extend to infinity. One field deforms in simple shear, the other in pure shear. For inclusions of infinite aspect ratio, we know (without having to resort to calculations) that the PDF of crystal orientations is uniquely determined by the strain ellipsoid (March 1932). Since both flow fields are described by spatially constant strain ellipsoids, it is always possible to find a pure strain field that corresponds to a given simple strain field (e.g. Jaeger et al. 2009). It seems likely that many more examples of non-uniqueness in the flow field can be found, especially when time-varying fields are taken into account. It is presently not known in practice whether the non-uniqueness of the solution places any significant limitations on the reconstruction of the velocity field based on observations of crystal orientations.

An additional potential difficulty in interpreting palaeo-flow directions from observations of crystal alignment arises because not all instances of crystal alignment are necessarily caused by differential rotation in shear flows. Crystal settling and compaction of cumulates may lead to fabrics that are indistinguishable from those caused by flow of magma (Holness et al. 2017). Similarly, SPO may form due to crystallization at the interface between mafic and felsic magma mushes (Pistone et al. 2015). At low melt concentrations, dislocation creep accompanied by recrystallization can form both SPO and CPO (crystallographic preferred orientation), thereby destroying some or all of the crystal alignment that was previously produced in the low-crystal concentration flow regime (Nicolas 1992). Detailed field observations may in the future be able to distinguish between the different types of SPO, but at present, no clear discriminating features have been identified (Holness et al. 2017).

\subsection{Beyond Jeffery's solution for dilute suspensions}

An important observation of crystal orientations in dykes is that the alignment is typically not perfectly parallel to the dyke walls. Instead, the dominant crystal orientation often is some $5-30^{\circ}$ towards the centre of the dyke relative to the flow direction (Blanchard et al. 1979; Knight \& Walker 1988; Arbaret et al. 1996). This pattern is sometimes described as an imbrication pattern or tiling (Den Tex 1969; Arbaret et al. 1996). The effect is greatest near the dyke walls and vanishes towards the centre (Knight \& Walker 1988). Furthermore, the angular deviation from the dyke walls appears to be greater for thicker dykes than for thinner dykes (Knight \& Walker 1988), although the intensity of the effect is often greater for thinner dykes (Geoffroy et al. 2002). Where observed, the imbrication pattern can be used to determine the palaeo-flow direction of magma in dykes (Knight \& Walker 1988; Geoffroy et al. 2002). However, this imbrication pattern is not predicted by Jeffery's 1922 solution for an isolated ellipsoid embedded in a viscous flow. A better understanding of the origin of the imbrication pattern is clearly desirable in order to have greater confidence in our present theory, or to amend the theory to fit these observations.

The behaviour of the crystal orientation PDF for Jeffery's solution in simple shear flow has been described by Bazargan et al. (2019). Whereas the individual crystals rotate continuously, but most slowly when aligned with the direction of shear, the PDF, if initialized as a random distribution of crystal orientations, oscillates between random and ordered states (Ježek et al. 1994; Marques \& Coelho 2003). At the same time, the peak of the distribution migrates in the direction of the solid body rotation of the flow. The greatest degree of orientation is obtained when $\theta=0$, that is, when the crystals mostly align with the direction of shear. An observed imbrication pattern is thus not necessarily an indication that Jeffery's theory is violated. However, as shown earlier, for strains corresponding to a few crystal rotations, small perturbations due to random variations in the flow conditions lead to a homogenization of the crystal orientations and hence a decrease in the intensity of the cyclic behaviour of the PDF. Consistent observations of imbrication patterns in dykes are thus strong evidence that the crystal rotations are described by dynamics that are, at least to some degree, different from Jeffery's theory for rigid ellipsoids.

Given the above considerations of the PDF evolution, one can conclude that the shape of the crystals does not have an effect on the location of the peak of the crystal orientation PDF because the rotation rate $\dot{\theta}(\theta)$ remains symmetric about $\theta=0$ (Willis 1977; Bazargan et al. 2019). The same argument holds true for non-linear rheologies (Bazargan et al. 2019). The velocity profile in dykes is described by Poiseuille flow (or so-called plug flow for power-law rheologies), but at any given, fixed position, the flow is locally identical with simple shear flow (Bazargan et al. 2019). The observed imbrication pattern in dykes is thus not a result of Poiseuille flow conditions in a dyke combined with Jeffery's solution. Furthermore, the observed imbrication patterns are typically fairly broad, indicating that the zone over which the velocity varies is significantly wider than the crystal size. We therefore do not believe that there is any strong shear localization which causes the crystal rotations to deviate from Jeffery's theory.

The most likely cause for departures from Jeffery's theory is a high volume concentration of crystals (e.g. Ildefonse et al. 1992b; Manga 1998). Jeffery's theory is exact for isolated crystals, and it is a good approximation for crystal concentrations corresponding to an intracrystal spacing of about one to two crystal lengths (Ildefonse et al. 1992b). For higher crystal concentrations, other theories are required which take into account the interactions between crystals caused by the local flow perturbations due to the crystal rotations. Significant advances have been made in the study of suspensions of long, cylindrical fibres. Three separate regimes have been identified: the dilute regime described by Jeffery's theory; the semidilute regime in the range $(D / L)^{2}<\phi<(D / L)$, where $\phi$ is the volume fraction of fibres, and $D$ and $L$ are the diameter and the length of 
the fibres, respectively; and the concentrated regime (Dinh \& Armstrong 1984; Doi \& Edwards 1988; Petrie 1999). The concentrated regime has received relatively little attention because the concentrations are so high that the fibres have to line up in parallel in order to allow significant fluid motion (Petrie 1999). If the same fundamentals apply to crystals with relatively low aspect ratio (with an equivalent value of $D / L$ of around 0.2 or greater), then the boundary between semi-dilute and concentrated regimes is significantly lower than the critical value of crystal concentrations in magmas beyond which the effective viscosity of the suspension abruptly increases and flow effectively ceases (Petford 2003). The concentrated regime is thus probably the most appropriate for magma flows just before solidification, but an unknown proportion of the final crystal alignment pattern may have formed in the semidilute regime that prevailed when the magma was somewhat hotter.

A common characteristic of the various theories of inclusion rotations in the semi-dilute regime is that rotations are not computed for individual inclusions, but instead the statistical effect of large numbers of particle-particle interactions is taken into account (Batchelor 1971; Dinh \& Armstrong 1984; Shaqfeh \& Fredrickson 1990; Petrie 1999). For example, the interactions may on average manifest themselves as diffusion of the orientation PDF in the angular directions, with possible anisotropy in the diffusion constant (Rahnama et al. 1995; Manga 1998). We briefly tested this concept with our model, but in a 2-D setting, there exists only one possible rotation direction, thus precluding anisotropy. For constant diffusion in the $\theta$-direction, the time-averaged peak of the PDF for simple shear flow remains in the flow direction. The observed imbrication pattern may require more complex theories, or perhaps the rotational behaviour of the crystals is inherently 3-D (i.e. the present 2-D analysis may be overly simplified). These concepts need to be tested further for magmatic systems using either experimental methods such as those of Arbaret et al. (2019), or numerical methods which solve explicitly for the combination of regional and crystal-induced flow (Yamato et al. 2011).

As a final note, we tested our model against the experimental results of Arbaret et al. (2019). For a final strain of $\gamma=2.0$, they find a peak angle of $\theta_{p}=2^{\circ}$ relative to the flow direction for isolated grains and $\theta=18^{\circ}$ for clustered grains. The overall fabric strength is characterized by an axial ratio of $R=1.479$. For the same conditions, using 1000 distinct PDF functions in order to approximate a lognormal distribution of crystal aspect ratios (with $\mu=0.5$ and $\sigma=0.85$ ), we find a peak angle of $\theta_{p}=20^{\circ}$ and a fabric strength corresponding to $R \approx 5$.6. While the correspondence between the model and the experimental data is relatively poor, the results illustrate that the experimental observations are well within the range of what is predicted by Jeffery's solution (although the observed differences between isolated and clustered grains indicate that Jeffery's theory is violated). It would be of fundamental interest to see whether the observed imbrication pattern persists at very large strains when the cyclic behaviour caused by the initial conditions has ceased (due to the continuous distribution of crystal aspect ratios) and the flow is thus more similar to that in a typical dyke.

\section{CONCLUSIONS}

We present numerical results for the magma velocity field and for the PDF of crystal orientations in common subvolcanic flow geometries.

(i) In channels such as dykes governed by Poiseuille flow, elongate crystals align with the flow direction. Where the channels abruptly change direction, the crystal orientations generally follow the channel geometry, but the pattern is smoothed out symmetrically over a distance of about one channel width from the axis of the bend. The degree of orientation displays some asymmetry about the axis of the bend, but it is not clear whether this effect is sufficiently strong to allow determination of the flow direction from field observations.

(ii) For magma exiting a dyke and entering a larger reservoir, we find that, away from the walls, elongate crystals preferentially align in the local direction of $\dot{\varepsilon}_{1}$, which is perpendicular to the flow direction at the dyke-reservoir transition. For the reverse flow of magma entering a dyke, both $\dot{\varepsilon}_{1}$ and the crystals align with the direction of the flow. This observation is in good agreement with results from analogue models.

(iii) In an inflating magma reservoir with uniform stretching of the bounding walls, crystals become aligned parallel to the walls throughout the domain except for the immediate vicinity of the inflow into the reservoir. For the reverse model of a deflating reservoir, the corresponding crystal orientations are radial and hence perpendicular to the walls.

(iv) Thermal convection causes crystal alignment parallel to the walls where the flow due to convection cells impinges on the boundary, and where flow is parallel to the boundaries. Crystal alignment is normal to the walls where the flow turns away from the boundary.

(v) In general, we find that identical flow regimes but with crystal inclusions of different aspect ratio lead to similar crystal alignment patterns. The overall degree of orientation is greater for crystals with higher aspect ratio. This result is fortuitous, as it means that a natural system with a range of crystal aspect ratios can be approximated by a model with a single aspect ratio, such as the mean of the aspect ratio distribution.

(vi) Our method solves the forward problem of finding preferred crystal orientations from the velocity field, and hence from the basic underlying dynamics. For typical applications, it is the inverse problem that needs to be solved, which consists of inferring the flow and its dynamics from observations of crystal orientations. An inherent difficulty of the inverse problem is non-uniqueness when isolated subdomains are considered. For example, boundary-parallel orientations may indicate boundary-parallel shear flow, or they may indicate pure shear flow impinging on the wall. For such cases, it is necessary to find a globally consistent flow field. On the other hand, crystal orientations normal to the boundary are probably good indicators of thermal convection, because the competing candidate, namely a local contraction of the boundary such as in a deflating reservoir, is physically unlikely to occur.

\section{DATA AVAILABILITY}

The data underlying this article will be shared on reasonable request to the corresponding author.

\section{REFER E N CES}

Arbaret, L., Diot, H. \& Bouchez, J.-L., 1996. Shape fabrics of particles in low concentration suspensions: 2D analogue experiments and application to tiling in magma, J. Struct. Geol., 18(7), 941-950.

Arbaret, L., Bystricky, M. \& Launeau, P., 2019. Crystal clustering in magmas: insights from HP-HT experiments, Comp. Rend. Geosci., 351(8), 574-585.

Batchelor, G.K., 1971. The stress generated in a non-dilute suspension of elongated particles by pure straining motion, J. Fluid Mech., 46, 813-829. Batchelor, G.K., 2000. An Introduction to Fluid Dynamics, Cambridge Univ. Press. 
Bazargan, M., Vachon, R. \& Hieronymus, C.F., 2019. Evolution of the statistical distribution of crystal orientations in time- and space-varying viscous flows, J. geophys. Int., 218(2), 773-786.

Blanchard, J.-P., Boyer, P. \& Gagny, C., 1979. Un nouveau critère de sens de mise en place dans une caisse filonienne: Le "pincement" des minéraux aux epontes: Orientation des minéraux dans un magma en écoulement, Tectonophysics, 53(1), 1-25.

Borradaile, G.J. \& Jackson, M., 2004. Anisotropy of magnetic susceptibility (AMS): magnetic petrofabrics of deformed rocks, Geol. Soc. Spec. Publ., 238, 299-360.

Buisson, C. \& Merle, O., 2002. Experiments on internal strain in lava dome cross sections, Bull. Volcanol., 64(6), 363-371.

Cañón-Tapia, E., 2004. Anisotropy of magnetic susceptibility of lava flows and dykes: a historical account, Geol. Soc. Lond. Spec. Publ., 238, 205225.

Cañón-Tapia, E. \& Chávez-Alvarez, J., 2004. Theoretical aspects of particle movement in flowing magma: implications for the anisotropy of magnetic susceptibility of dykes, Geol. Soc., Lond., Spec. Publ., 238(1), 227-249.

Chistyakova, S. \& Latypov, R., 2010. On the development of internal chemical zonation in small mafic dykes, Geol. Mag., 147(1), 1-12.

Clemens, J.D., 2003. S-type granitic magmas-petrogenetic issues, models and evidence, Earth-Sci. Rev., 61(1), 1-18.

Cnudde, V., Boone, M., Dewanckele, J., Dierick, M., Hoorebeke, L.V. \& Jacobs, P., 2011. 3d characterization of sandstone by means of X-ray computed tomography, Geosphere, 7(1), 54-61.

Comsol Multiphysics $囚$, 2017. v5.3, Comsol AB, Stockholm, Sweden.

Costa, A., Caricchi, L. \& Bagdassarov, N., 2009. A model for the rheology of particle-bearing suspensions and partially molten rocks, J. geophys. Int., 10(3), doi:10.1029/2008GC002138.

Den Tex, E., 1969. Origin of ultramafic rocks, their tectonic setting and history: a contribution to the discussion of the paper "the origin of ultramafic and ultrabasic rocks" by P.J. Wyllie, Tectonophysics, 7(5), 457-488.

Dinh, S.M. \& Armstrong, R.C., 1984. A rheological equation of state for semiconcentrated fiber suspensions, J. Rheol., 28(3), 207-227.

Doi, M. \& Edwards, S.F., 1988. The Theory of Polymer Dynamics, International Series of Monographs on Physics, Oxford Univ. Press.

Eriksson, P.I., Riishuus, M.S., Sigmundsson, F. \& Elming, S.-R., 2011. Magma flow directions inferred from field evidence and magnetic fabric studies of the Streitishvarf composite dike in east Iceland, J. Volc. Geotherm. Res., 206(1), 30-45.

Fisher, N.I., 1995. Statistical Analysis of Circular Data, Cambridge Univ. Press.

Freeman, B., 1985. The motion of rigid ellipsoidal particles in slow flows, Tectonophysics, 113(1), 163-183.

Fries, J., Einarsson, J. \& Mehlig, B., 2017. Angular dynamics of small crystals in viscous flow, Phys. Rev. Fluids, 2(1), doi:10.1103/PhysRevFluids.2.014302.

Galland, O., 2018. Storage and transport of magma in the layered crustformation of sills and related flat-lying intrusions, in Volcanic and Igneous Plumbing Systems, Chapter 5, pp. 113-138, ed. Burchardt, S., Elsevier.

Geoffroy, L., Callot, J.P., Aubourg, C. \& Moreira, M., 2002. Magnetic and plagioclase linear fabric discrepancy in dykes: a new way to define the flow vector using magnetic foliation, Terra Nova, 14(3), 183-190.

Giordano, D., Russell, J.K. \& Dingwell, D.B., 2008. Viscosity of magmatic liquids: A model, Earth planet. Sci. Lett., 271(1), 123-134.

Gudmundsson, A., 2012. Magma chambers: Formation, local stresses, excess pressures, and compartments, J. Volc. Geotherm. Res., 237-238, $19-41$.

Gutiérrez, F., Payacán, I., Gelman, S.E., Bachmann, O. \& Parada, M.A., 2013. Late-stage magma flow in a shallow felsic reservoir: merging the anisotropy of magnetic susceptibility record with numerical simulations in La Gloria Pluton, central Chile, J. geophys. Res., 118(5), 1984-1998.

Henry, B., 1997. The magnetic zone axis: a new element of magnetic fabric for the interpretation of the magnetic lineation, Tectonophysics, 271(3), 325-331.

Holness, M.B., Vukmanovic, Z. \& Mariani, E., 2017. Assessing the role of compaction in the formation of adcumulates: a microstructural perspective, J. Petrol., 58(4), 643-673.
Horsman, E., Tikoff, B. \& Morgan, S., 2005. Emplacement-related fabric and multiple sheets in the Maiden Creek sill, Henry Mountains, Utah, USA, J. Struct. Geol., 27(8), 1426-1444.

Ildefonse, B., Launeau, P., Bouchez, J.-L. \& Fernandez, A., 1992a. Effect of mechanical interactions on the development of shape preferred orientations: a two-dimensional experimental approach, J. Struct. Geol., 14(1), 73-83.

Ildefonse, B., Sokoutis, D. \& Mancktelow, N.S., 1992b. Mechanical interactions between rigid particles in a deforming ductile matrix. Analogue experiments in simple shear flow, J. Struct. Geol., 14(10), 1253-1266.

Jaeger, J.C., Cook, N. G.W. \& Zimmerman, R., 2009. Fundamentals of Rock Mechanics, John Wiley \& Sons.

Ježek, J., Melka, R., Schulmann, K. \& Venera, Z., 1994. The behaviour of rigid triaxial ellipsoidal particles in viscous flows-modeling of fabric evolution in a multiparticle system, Tectonophysics, 229(3), 165-180.

Ježek, J., Schulmann, K. \& Segeth, K., 1996. Fabric evolution of rigid inclusions during mixed coaxial and simple shear flows, Tectonophysics, 257(2), 203-221.

Jeffery, G.B., 1922. The motion of ellipsoidal particles immersed in a viscous fluid, Proc. R. Soc. Lond. A, 102(715), 161-179.

Jiang, D., 2007. Numerical modeling of the motion of rigid ellipsoidal objects in slow viscous flows: a new approach, J. Struct. Geol., 29(2), 189-200.

Jiang, D., 2012. A general approach for modeling the motion of rigid and deformable ellipsoids in ductile flows, Comput. Geosci., 38(1), 52-61.

Khan, M.A., 1962. The anisotropy of magnetic susceptibility of some igneous and metamorphic rocks, J. geophys. Res., 67(7), 2873-2885.

Knight, M.D. \& Walker, G.P.L., 1988. Magma flow directions in dikes of the Koolau Complex, Oahu, determined from magnetic fabric studies, $J$. geophys. Res., 93(B5), 4301-4319.

Kratinová, Z., Závada, P., Hrouda, F. \& Schulmann, K., 2006. Non-scaled analogue modelling of AMS development during viscous flow: a simulation on diapir-like structures, Tectonophysics, 418(1), 51-61.

Kumar Samanta, S., Mandal, N. \& Chakraborty, C., 2003. Flow patterns around rigid inclusions in a multiple inclusion system undergoing bulk simple shear deformation, J. Struct. Geol., 25(2), 209-221.

Launeau, P. \& Cruden, A.R., 1998. Magmatic fabric acquisition mechanisms in a syenite: Results of a combined anisotropy of magnetic susceptibility and image analysis study, J. geophys. Res., 103(B3), 5067-5089.

Launeau, P. \& Robin, P.Y.F., 1996. Fabric analysis using the intercept method, Tectonophysics, 267(1), 91-119.

Mandal, N., Samanta, S.K., Bhattacharyya, G. \& Chakraborty, C., 2003. Deformation of ductile inclusions in a multiple inclusion system in pure shear, J. Struct. Geol., 25(9), 1359-1370.

Manga, M., 1998. Orientation distribution of microlites in obsidian, J. Volc. Geotherm. Res., 86(1), 107-115.

March, A., 1932. Mathematische theorie der regelung nach der korngestah bei affiner deformation, Zeitschrift für Kristallographie - Crystalline materials, 81(1-6), 285-297.

Marques, F.O. \& Coelho, S., 2003. 2-D shape preferred orientations of rigid particles in transtensional viscous flow, J. Struct. Geol., 25(6), 841-854.

Marques, F.O., Taborda, R.M. \& Antunes, J.V., 2005. 2D rotation of rigid inclusions in confined bulk simple shear flow: a numerical study, J. Struct. Geol., 27(12), 2171-2180.

Marques, F.O., Mandal, N., Taborda, R., Antunes, J.V. \& Bose, S., 2014. The behaviour of deformable and non-deformable inclusions in viscous flow, Earth-Sci. Rev., 134, 16-69.

Marsh, B.D., 1989. On convective style and vigor in sheet-like magma chambers, J. Petrol., 30(3), 479-530.

Mattsson, T., Burchardt, S., Almqvist, B. S.G. \& Ronchin, E., 2018. Syn-emplacement fracturing in the Sandfell laccolith, eastern IcelandImplications for rhyolite intrusion growth and volcanic hazards, Front. Earth Sci., 6, doi:10.3389/feart.2018.00005.

Montgomery-Brown, E.K., Sinnett, D., Poland, M., Segall, P., Orr, T., Zebker, H. \& Miklius, A., 2010. Geodetic evidence for en echelon dike emplacement and concurrent slow slip during the June 2007 intrusion and eruption at Killauea volcano, Hawaii, J. geophys. Res., 115(B7), doi:10.1029/2009JB006658. 
Nicolas, A., 1992. Kinematics in magmatic rocks with special reference to gabbros, J. Petrol., 33(4), 891-915.

Nobile, A., Pagli, C., Keir, D., Wright, T.J., Ayele, A., Ruch, J. \& Acocella, V., 2012. Dike-fault interaction during the 2004 Dallol intrusion at the northern edge of the Erta Ale Ridge (Afar, Ethiopia), Geophys. Res. Lett., 39(19), doi:10.1029/2012GL053152.

Pallister, J.S., et al., 2010. Broad accommodation of rift-related extension recorded by dyke intrusion in Saudi Arabia, Nat. Geosci., 3(10), 705-712.

Palmer, H.C., Ernst, R.E. \& Buchan, K.L., 2007. Magnetic fabric studies of the Nipissing sill province and Senneterre dykes, Canadian Shield, and implications for emplacement, Can. J. Earth Sci., 44(4), 507-528.

Passchier, C.W., 1987. Stable positions of rigid objects in non-coaxial flow: a study in vorticity analysis, J. Struct. Geol., 9(5), 679-690.

Paterson, S.R., 2009. Magmatic tubes, pipes, troughs, diapirs, and plumes: late-stage convective instabilities resulting in compositional diversity and permeable networks in crystal-rich magmas of the Tuolumne batholith, Sierra Nevada, California, Geosphere, 5(6), 496-527.

Paterson, S.R., Fowler, T.K., Schmidt, K.L., Yoshinobu, A.S., Yuan, E.S. \& Miller, R.B., 1998. Interpreting magmatic fabric patterns in plutons, Lithos, 44(1), 53-82.

Payacán, I., Gutiérrez, F., Gelman, S.E., Bachmann, O. \& Parada, M.A., 2014. Comparing magnetic and magmatic fabrics to constrain the magma flow record in La Gloria pluton, central Chile, J. Struct. Geol., 69, 32-46.

Petford, N., 2003. Rheology of granitic magmas during ascent and emplacement, Ann. Rev. Earth planet. Sci., 31(1), 399-427.

Petri, B., Almqvist, B.S. \& Pistone, M., 2020. 3d rock fabric analysis using micro-tomography: An introduction to the open-source tomofab matlab code, Comput. Geosci., 138, 104444.

Petrie, C.J.S., 1999. The rheology of fibre suspensions, J. Non-Newton. Fluid Mech., 87(2), 369-402.

Pistone, M., Blundy, J. \& Brooker, R., 2015. Textural and chemical consequences of interaction between hydrous mafic and felsic magmas: an experimental study, Contrib. Mineral. Petrol., 171, doi:10.1007/s00410015-1218-4.

Pons, J., Barbey, P., Nachit, H. \& Burg, J.P., 2006. Development of igneous layering during growth of Pluton: the Tarçouate Laccolith (Morocco), Tectonophysics, 413(3), 271-286.

Rahnama, M., Koch, D.L. \& Shaqfeh, E. S.G., 1995. The effect of hydrodynamic interactions on the orientation distribution in a fiber suspension subject to simple shear flow, Phys. Fluids, 7(3), 487-506.

Reed, L.J. \& Tryggvason, E., 1974. Preferred orientations of rigid particles in a viscous matrix deformed by pure shear and simple shear, Tectonophysics, 24(1), 85-98.

Shaqfeh, E.S.G. \& Fredrickson, G.H., 1990. The hydrodynamic stress in a suspension of rods, Phys. Fluids A: Fluid Dyn., 2(1), 7-24.

Silva, P., Marques, F., Miranda, J., Henry, B. \& Mateus, A., 2001. Anisotropy of magnetic susceptibility constraints on variscan obduction processes in the bragança massif (ne portugal), Tectonophysics, 341(1), 95-119.
Silva, P., Marques, F., Henry, B., Mateus, A., Lourenço, N. \& Miranda, J., 2004. Preliminary results of a study of magnetic properties in the foumzguid dyke (Morocco), Phys. Chem. Earth, A/B/C, 29(13), 909-920.

Silva, P., Marques, F., Machek, M., Henry, B., Hirt, A., Roxerová, Z., Madureira, P. \& Vratislav, S., 2014. Evidence for non-coaxiality of ferrimagnetic and paramagnetic fabrics, developed during magma flow and cooling in a thick mafic dyke, Tectonophysics, 629, 155-164.

Silva, P.F., Marques, F.O., Henry, B., Madureira, P., Hirt, A.M., Font, E. \& Lourenço, N., 2010. Thick dyke emplacement and internal flow: a structural and magnetic fabric study of the deep-seated dolerite dyke of foum zguid (southern Morocco), J. geophys. Res., 115(B12), doi:10.1029/2010JB007638.

Smith, J.V., 2002. Structural analysis of flow-related textures in lavas, EarthSci. Rev., 57(3), 279-297.

Szeri, A.J., 1993. Pattern formation in recirculating flows of suspensions of orientable particles, Philos. Trans.: Phys. Sci. Eng., 345(1677), 477-506.

Tarling, D. \& Hrouda, F., 1993. Magnetic Anisotropy of Rocks, Springer Netherlands.

Tauxe, L., Gee, J.S. \& Staudigel, H., 1998. Flow directions in dikes from anisotropy of magnetic susceptibility data: the bootstrap way, J. geophys. Res., 103(B8), 17 775-17 790.

Trebbin, M., Steinhauser, D., Perlich, J., Buffet, A., Roth, S.V., Zimmermann, W., Thiele, J. \& Förster, S., 2013. Anisotropic particles align perpendicular to the flow direction in narrow microchannels, Proc. Natl. Acad. Sci. U.S.A., 110(17), 6706-6711.

Truesdell, C., 1953. The physical components of vectors and tensors, $J$. Appl. Math. Mech., 33(10-11), 345-356.

Turcotte, D. \& Schubert, G., 2014. Geodynamics, Cambridge Univ. Press.

Vernon, R.H. \& Paterson, S.R., 2006. Mesoscopic structures resulting from crystal accumulation and melt movement in granites, Earth Environ. Sci. Trans. R. Soc. Edinburgh, 97(4), 369-381.

Willis, D.G., 1977. A kinematic model of preferred orientation, GSA Bull., 88(6), 883-894.

Yamato, P., Tartese, R., Duretz, T. \& May, D., 2011. Numerical modelling of magma transport in dykes, Tectonophysics, 526-529, 97-109.

Yun, S., Segall, P. \& Zebker, H., 2006. Constraints on magma chamber geometry at Sierra Negra Volcano, Galápagos Islands, based on InSAR observations, J. Volc. Geotherm. Res., 150(1), 232-243.

Závada, P., Kratinová, Z., Kusbach, V. \& Schulmann, K., 2009. Internal fabric development in complex lava domes, Tectonophysics, 466(1), 101113.

Závada, P., Dědeček, P., Lexa, J. \& Keller, G.R., 2015. Devils Tower (Wyoming, USA): a lava coulée emplaced into a maar-diatreme volcano? Geosphere, 11(2), 354-375.

Zucali, M., Voltolini, M., Ouladdiaf, B., Mancini, L. \& Chateigner, D., 2014. The $3 \mathrm{~d}$ quantitative lattice and shape preferred orientation of a mylonitised metagranite from Monte Rosa (Western Alps): combining neutron diffraction texture analysis and synchrotron X-ray microtomography, $J$. Struct. Geol., 63, 91-105. 